\title{
ON THE STRUCTURE OF LARGE SUM-FREE SETS OF INTEGERS
}

\author{
TUAN TRAN
}

\begin{abstract}
A set of integers is called sum-free if it contains no triple $(x, y, z)$ of not necessarily distinct elements with $x+y=z$. In this paper, we provide a structural characterisation of sum-free subsets of $\{1,2, \ldots, n\}$ of density at least $2 / 5-c$, where $c$ is an absolute positive constant. As an application, we derive a stability version of Hu's Theorem [Proc. Amer. Math. Soc. 80 (1980), 711-712] about the maximum size of a union of two sum-free sets in $\{1,2, \ldots, n\}$. We then use this result to show that the number of subsets of $\{1,2, \ldots, n\}$ which can be partitioned into two sum-free sets is $\Theta\left(2^{4 n / 5}\right)$, confirming a conjecture of Hancock, Staden and Treglown arXiv:1701.04754.
\end{abstract}

\section{INTRODUCTION}

A triple $(x, y, z)$ of not necessarily distinct integers is called a Schur triple if $x+y=z$. Given a positive integer $r$, we say that a subset $A$ of $[n]:=\{1,2, \ldots, n\}$ is $r$-wise sumfree if there exists an $r$-colouring of $A$ which contains no monochromatic Schur triples. When $r=1$, we simply call such sets sum-free. Here we derive a structural theorem for large sum-free sets, and apply it to prove a sharp bound, up to a constant factor, on the number of 2 -wise sum-free subsets of $[n]$. In the following subsections we will review what is already known before presenting our results.

1.1. Sum-free sets and their structure. A natural extremal question, which was asked by Abbott and Wang [1] in 1977, is how large an $r$-wise sum-free subset of $[n]$ can be. We denote the maximum by $\mu(n, r)$. It is not difficult to see that $\mu(n, 1)=n-\lfloor n / 2\rfloor$, and this bound is attained by the set of odd numbers in $[n]$ and by the interval $\{\lfloor n / 2\rfloor+1, \ldots, n\}$. The following definition helps motivate the study of $\mu(n, r)$ for $r \geq 2$. Let $h(r)$ denote the largest positive integer $m$ for which there exists some way of partitioning $[m]$ into $r$ sets that are sum-free modulo $m+1$. For example, one has $h(2)=4, h(3)=13$ and $h(4)=44$ (see [1, Problem I]). Abbot and Wang [1] showed

$$
\mu(n, r) \geq n-\left\lfloor\frac{n}{h(r)+1}\right\rfloor
$$

for every integer $r \geq 2$, and conjectured that the equality holds. In 1980, $\mathrm{Hu}$ [32] provided a short and elegant proof of this conjecture for $r=2$, that is, $\mu(n, 2)=$ $n-\lfloor n / 5\rfloor$. To see why $\mu(n, 2) \geq n-\lfloor n / 5\rfloor$, one can consider the set $\{a \in[n]: a \equiv$ $1,4(\bmod 5)\} \cup\{b \in[n]: b \equiv 2,3(\bmod 5)\}$. For $r \geq 3$, though there are several interesting general upper bounds for $\mu(n, r)$ (see [1, 30]), none of them matches the lower bound given by Abbot and Wang.

Given the extremal result, great efforts has been made to better understand the general structure of large sum-free sets in $[n]$. The earliest result in this direction was obtained by Freiman [25] who showed that, loosely speaking, a sum-free set of density greater than 5/12 is 'interval like' or consists entirely of odd numbers. 
Theorem 1.1 (Freiman). Every sum-free subset $A$ of $[n]$ with $|A| \geq 5 n / 12+2$ satisfies one of the following conditions:

(i) A consists of odd numbers;

(ii) the minimum element of $A$ is at least $|A|$.

In an unpublished note, Deshouillers, Freiman and Sós proved that the conclusion of Theorem 1.1 continues to hold when $5 n / 12+2$ is replaced by $2 n / 5+1$. The following examples show that the condition $|A| \geq 2 n / 5+1$ cannot be relaxed. Indeed, supposing that $n$ is divisible by 5 , we consider the sets $A_{1}=\{a \in[n]: a \equiv 1,4(\bmod 5)\}$, $A_{2}=\{a \in[n]: a \equiv 2,3(\bmod 5)\}$, and $A_{3}=\{n / 5+1, \ldots, 2 n / 5\} \cup\{4 n / 5+1, \ldots, n\}$. We can see that each $A_{i}$ is a sum-free subset of $[n]$ of size $2 n / 5$, and that they are very far from satisfying property (i) or (ii) from Theorem 1.1 .

A few years later, Deshouillers, Freiman, Sós and Temkin 15 succeeded in slightly breaking the $2 n / 5$ barrier (see Theorem 1.2 below). Roughly speaking, they proved that the structure of a sum-free set in $[n]$ of size greater than $2 n / 5-O(1)$ is described by Theorem [1.1, or close to one of the sets $A_{i}$ mentioned previously 1

Theorem 1.2 (Deshouillers-Freiman-Sós-Temkin). For every $x>0$, there exist numbers $n_{0} \in \mathbb{N}$ and $K>0$ such that whenever $A$ is a sum-free set in $[n]$ satisfying $n \geq n_{0}$ and $|A| \geq 2 n / 5-x$, then $A$ has one of the following properties:

(i) all the elements of $A$ are odd;

(ii) all the elements of $A$ are congruent to 1 or 4 modulo 5;

(iii) all the elements of $A$ are congruent to 2 or 3 modulo 5;

(iv) the minimum element of $A$ is greater than or equal to $|A|$;

(v) $A$ is contained in $\left[\frac{n}{5}-K, \frac{2 n}{5}+K\right] \cup\left[\frac{4 n}{5}-K, n\right]$.

Besides being interesting in their own right, these results have found several applications (see [38, 26, 8, 9]). We remark that very few structural results are known for large sum-free sets in finite abelian groups, cf. [13, 28, 35, 14, 36, 16, 5].

1.2. Counting sum-free sets. Let $\mathrm{SF}_{r}(n)$ denote the collection of $r$-wise sum-free subsets of $[n]$. By considering all possible subsets of the set $\{\lfloor n / 2\rfloor+1, \ldots, n\}$, we see that $[n]$ contains at least $2^{n / 2}$ sum-free sets. Cameron and Erdős [12] in 1990 conjectured that this trivial lower bound is within a constant factor of the truth, that is, $\left|\mathrm{SF}_{1}(n)\right|=O\left(2^{n / 2}\right)$. Their conjecture resisted various attempts at proof for over ten years [2, 11, 25], until it was confirmed independently by Green [26] and Sapozhenko [41. In fact, they proved that there are asymptotically $c(n) 2^{n / 2}$ such sets, where $c(n)$ takes two different constant values depending on the parity of $n$. Recently, a refinement of the Cameron-Erdös conjecture was obtained by Alon, Balogh, Morris and Samotij [4], giving an upper bound on the number of sum-free sets in $[n]$ of size $s$, for all $s \in\{1,2, \ldots,\lceil n / 2\rceil\}$.

For $r=2$, recall that the set $\{a \in[n]: a \equiv 1,4(\bmod 5)\} \cup\{b \in[n]: b \equiv 2,3(\bmod 5)\}$ is 2-wise sum-free, and so are all of its subsets, giving $\left|\mathrm{SF}_{2}(n)\right| \geq 2^{4 n / 5}$. Inspired by [41, 26], Hancock, Staden and Treglown [30] considered this counting problem, among other things, and conjectured that this simple bound is in fact the correct estimate on $\left|\mathrm{SF}_{2}(n)\right|$. Thus they put forward the following conjecture.

Conjecture 1.3 (Hancock-Staden-Treglown). $\left|\mathrm{SF}_{2}(n)\right|=O\left(2^{4 n / 5}\right)$.

\footnotetext{
${ }^{1}$ Their result provides no information about sum-free sets in $[n]$ of size less than $2 n / 5-\sqrt{n}$.
} 
Note that Hancock et al. applied the container theorems of Balogh, Morris and Samotij [10], and Saxton and Thomason [42], to establish $\left|\mathrm{SF}_{2}(n)\right|=2^{4 n / 5+o(n)}$. We recommend [30, 31] and the references therein for related results concerning $\mathcal{L}$-free subsets of $[n]$, where $\mathcal{L}$ is a homogeneous system of linear equations.

1.3. Our results. Here we go one step beyond Theorem 1.2, and provide a structural characterisation of sum-free sets of size greater than $(2 / 5-c) n$, where $c$ is an absolute positive constant.

Theorem 1.4. There exists an absolute positive constant $c$ so that the following holds for every $n \in \mathbb{N}$ and every $\eta \in \mathbb{R}$ with $2 / n \leq \eta \leq c$. Let $A$ be a sum-free subset of $[n]$ with $|A| \geq(2 / 5-\eta) n$. Then one of the following alternatives occurs:

(i) all the elements of $A$ are odd;

(ii) all the elements of $A$ are congruent to 1 or 4 modulo 5;

(iii) all the elements of $A$ are congruent to 2 or 3 modulo 5;

(iv) the minimum element of $A$ is greater than or equal to $|A|$;

(v) $A$ is contained in $\left[\left(\frac{1}{5}-200 \sqrt{\eta}\right) n,\left(\frac{2}{5}+200 \sqrt{\eta}\right) n\right] \cup\left[\left(\frac{4}{5}-200 \sqrt{\eta}\right) n, n\right]$.

Note that there are sum-free subsets of $[n]$ of density $3 / 8$ structurally different from those appeared in the above theorem, such as $\{a \in[n]: a \equiv 3,4,5(\bmod 8)\}$ and $\{a \in[n]: a \equiv 4,5,6(\bmod 8)\}$. As an application of Theorem 1.4, we derive a stability version of Hu's result (Proposition 3.2), which may be of independent interest.

The proof of Theorem 1.4 draws on a number of ideas from [15]. In particular, as in [15] we make use of an inverse theorem of Lev and Smeliansky [37] for subsets of integers with small difference set. We also develop a number of new ideas in order to deal with the case when the smallest element of $A$ is sublinear in $n$, thereby making the argument substantially more involved.

The second part of the paper deals with Conjecture 1.3. We show

$$
\left|\mathrm{SF}_{2}(n)\right|=O\left(2^{4 n / 5}\right),
$$

settling the conjecture in the affirmative.

Theorem 1.5. The number of 2-wise sum-free subsets of $[n]$ is $O\left(2^{4 n / 5}\right)$.

The proof technique is inspired by the methods of [12, 26, 4, 3, 9]. Among other tools we use a container lemma of Hancock et al. [30], an arithmetic removal lemma of Green [27, our stability version of Hu's theorem, and a recent bound on the number of sets of integers with small sumset due to Green and Morris [29].

1.4. Organisation and notation. The rest is organised as follows. Section 2 is devoted to the study of large sum-free subsets of $[n]$. In Section 2.1 we provide the main lemmas and use them to obtain Theorem 1.4. We collect together some useful results in Section 2.2 and prove the main lemmas in Sections 2.3, 2.4 and 2.5. Section 3 deals with the enumerating problem. In Section 3.1, we outline the proof of Theorem 1.5, We present the main tools in Section 3.2 and prove Theorem 1.5 in Section 3.3. We close, in Section 4, with some remarks and open problems.

Given two non-empty sets $A, B \subset \mathbb{Z}$, we define

$$
A+B:=\{a+b: a \in A, b \in B\} \text { and } A-B:=\{a-b: a \in A, b \in B\}
$$

to be their sumset and difference set, respectively. For repeated addition we write $k A$ for the $k$-fold sumset $A+\ldots+A$, in contrast to $k \cdot A:=\{k a: a \in A\}$. For a 
finite set $A$ of integers, denote by $\min (A)$ and $\max (A)$ the minimum and maximum elements of $A$ respectively, and let $\ell(A):=\max (A)-\min (A)+1$. Let $A_{+}$stands for the set $\{a \in A: a>0\}$. The greatest common divisor of all the elements in $A-A$ will be denoted by $d(A)$. We denote by $E$ the set of all even and by $O$ the set of all odd numbers in $[n]$; the value of $n$ will always be clear from the context. Denote

$$
F_{1,4}=\{a \in[n]: a \equiv 1,4(\bmod 5)\} \text { and } F_{2,3}=\{a \in[n]: a \equiv 2,3(\bmod 5)\} .
$$

For real numbers $\alpha$ and $\beta$, we employ the interval notation

$$
[\alpha, \beta]:=\{x \in \mathbb{Z}: \alpha \leq x \leq \beta\},
$$

and similarly for open intervals. Throughout the paper we omit floor and ceiling signs where the argument is unaffected.

\section{LARGE SUM-FREE SETS}

2.1. Main lemmas and a proof of Theorem 1.4. Here we state three main lemmas and explain how to obtain Theorem 1.4 from them. In Lemma 2.1, we deal with the sum-free sets $A$ for which the ratio $\frac{\min (A)}{\max (A)}$ is large. In Lemma 2.2, we deal with the case when the ratio $\frac{\min (A)}{\max (A)}$ is neither too large nor too small. Lemmas 2.1 and 2.2 follow closely the approach from [15], and only minor adaptations are needed in our setting. Finally in Lemma 2.3, which is much more delicate, we study the case that the ratio $\frac{\min (A)}{\max (A)}$ is small. The methods used in [15] do not seem to adapt easily to this case, so we have been forced to devise our own arguments.

Our first main lemma, proven in Section 2.3, says that if the ratio $\frac{\min (A)}{\max (A)}$ is large then $A$ satisfies condition $(v)$ from Theorem 1.4.

Lemma 2.1 (Large range). Let $1 / n \leq \eta \leq 1 / 160^{2}$, and let $A$ be a sum-free subset of $[n]$ such that $n \in A, d(A)=1,|A| \geq(2 / 5-\eta) n$, and

$$
(1 / 5-\sqrt{\eta}) n \leq \min (A)<|A| \text {. }
$$

Then $A$ is contained in $\left[\left(\frac{1}{5}-\sqrt{\eta}\right) n,\left(\frac{2}{5}+32 \sqrt{\eta}\right) n\right] \cup\left[\left(\frac{4}{5}-31 \sqrt{\eta}\right) n, n\right]$.

Our second main lemma rules out the possibility that the ratio $\frac{\min (A)}{\max (A)}$ is neither too large nor too small. We provide the proof in Section 2.4.

Lemma 2.2. Let $1 / n \leq \eta \leq 1 / 175^{2}$, and let $A$ be a sum-free subset of $[n]$ such that $n \in A, d(A)=1$, and

Then $|A| \leq(2 / 5-2 \eta) n$.

$$
35 \sqrt{\eta} n \leq \min (A) \leq(1 / 5-\sqrt{\eta}) n .
$$

Our third and final main lemma, proven in Section 2.5, states that if $\min (A)$ is small compared to $\max (A)$ then $A$ satisfies condition (i), (ii) or (iii) from Theorem 1.4 .

Lemma 2.3 (Small range). There exists an absolute positive constant $c$ such that the following holds for every $n \in \mathbb{N}$ and every $\eta \in \mathbb{R}$ with $1 / n \leq \eta \leq c$. Let $A$ be a sum-free subset of $[n]$ satisfying $A \cap E \neq \emptyset,|A| \geq(2 / 5-\eta) n$, and

$$
\min (A) \leq 35 \sqrt{\eta} n .
$$

Then $A$ is contained in either $F_{1,4}$ or $F_{2,3}$.

With these lemmas in hand, we can prove Theorem 1.4. 
Proof of Theorem 1.4. Set $c=\min \left\{q 2.3, \frac{1}{175^{2}}\right\}$, where $q_{2.3}$ is the absolute positive constant from Lemma 2.3. Denote by $m$ and $N$ the minimum and maximum elements of $A$ respectively. We may assume without restriction of generality that $A \cap E \neq \emptyset$ and $\min (A)<|A|$, that is, $A$ does not satisfy properties (i) and (iv). In order to apply the main lemmas, we must show that $d(A)=1$ and $\eta \geq 1 / N$. Suppose to the contrary that $d(A)>1$. Then there are two possibilities: either $d(A)=2$ or $d(A) \geq 3$. In the later case, we clearly have $|A| \leq n / 3+1$. In the former case, since $A \cap E \neq \emptyset$, $A$ consists of even numbers. In particular, the set $\{a / 2: a \in A\}$ is a sum-free subset of $[n / 2]$, and so $|A| \leq n / 4+1$. In either case, we always have $|A| \leq n / 3+1$, which contradicts the assumptions that $|A| \geq(2 / 5-\eta) n$ and $1 / n \leq \eta \leq 1 / 175^{2}$. To verify the inequality $\eta \geq 1 / N$, we note that $|A| \leq N / 2+1$ as $A$ is a sum-free subset of $[N]$. Since $|A| \geq(2 / 5-\eta) n$, this implies $N \geq n / 2$ when $2 / n \leq \eta \leq 1 / 175^{2}$, giving the required bound $\eta \geq 2 / n \geq 1 / N$.

The proof now falls naturally into three cases:
(a) $(1 / 5-\sqrt{\eta}) N \leq m<|A|$
(b) $35 \sqrt{\eta} N \leq m \leq(1 / 5-\sqrt{\eta}) N$
(c) $m \leq 35 \sqrt{\eta} N$

We can easily rule out case $(b)$ using Lemma 2.2. If case $(c)$ occurs then Lemma 2.3 would imply that $A$ is a subset of either $F_{1,4}$ or $F_{2,3}$. Finally we deal with case $(a)$. We may apply Lemma 2.1 to conclude $A \subseteq\left[\left(\frac{1}{5}-\sqrt{\eta}\right) N,\left(\frac{2}{5}+32 \sqrt{\eta}\right) N\right] \cup\left[\left(\frac{4}{5}-31 \sqrt{\eta}\right) N, N\right]$. In particular, we have $|A| \leq(2 / 5+65 \sqrt{\eta}) N$. This upper bound on $|A|$, in conjunction with the assumption that $|A| \geq(2 / 5-\eta) n$, shows $N \geq(1-163 \sqrt{\eta}) n$, which in turn implies $A \subseteq\left[\left(\frac{1}{5}-200 \sqrt{\eta}\right) n,\left(\frac{2}{5}+200 \sqrt{\eta}\right) n\right] \cup\left[\left(\frac{4}{5}-200 \sqrt{\eta}\right) n, n\right]$ when $\eta \leq 1 / 175^{2}$.

2.2. Inverse theorems. Here we collect together a number of inverse theorems that are essential for proving the main lemmas.

Sets with small sumset are a central object of interest in Arithmetic Combinatorics and have been extensively studied in recent years (see, for example, [43]). One of the main results in this area is Freiman's inverse theorem [23] which states that if $A \subset \mathbb{Z}$ and $|A+A| \leq K|A|$ for some fixed $K$, then $A$ is a dense subset of a generalised arithmetic progression of bounded rank. In fact, the statement still holds in a slightly more general situation, when one considers $A+B$ instead of $A+A$. This was shown by Ruzsa [40].

For relatively small $K$, one can obtain more precise information, which plays a crucial role in our study. It is not hard to see that for any finite and non-empty sets $A, B \subset \mathbb{Z}$, one has

$$
|A+B| \geq|A|+|B|-1
$$

with equality if and only if $A$ and $B$ are arithmetic progressions with the same step. There has been much on generalising this result. For instance, Lev and Smeliansky [37] proved the following theorem.

Lemma 2.4 (Lev-Smeliansky). Let $A$ and $B$ be two finite sets of integers such that $|A+B| \leq|A|+|B|+\min (|A|,|B|)-4$. Then $A$ is contained in an arithmetic progression of length $|A+B|-|B|+1$ and $B$ is contained in an arithmetic progression of length $|A+B|-|A|+1$, where both progressions have the same step.

The special case of the above result for $A=B$ is the famous Freiman's $3 k-4$ theorem 23]. For our investigation we shall, however, need a "difference version" of this theorem, which follows readily from Lemma 2.4 . 
Lemma 2.5. Let $A$ be a finite set in $\mathbb{Z}$ such that $d(A)=1$. Then

$$
\left|(A-A)_{+}\right| \geq \min \left\{\frac{1}{2}(|A|+\ell(A)-2), \frac{3}{2}|A|-2\right\} .
$$

Proof. Suppose for a contradiction that $\left|(A-A)_{+}\right|<\min \left\{\frac{1}{2}(|A|+\ell(A)-2), \frac{3}{2}|A|-2\right\}$. As $\left|(A-A)_{+}\right| \geq \frac{1}{2}|A-A|-\frac{1}{2}$, it follows that $|A-A| \leq \min \{|A|+\ell(A)-2,3|A|-4\}$. By Lemma 2.4, we learn that $A$ is contained in an arithmetic progression of length $|A-A|-|A|+1 \leq \ell(A)-1$. This implies $d(A)>1$, a contradiction.

To our knowledge, the only extension of the $3 k-4$ Theorem that applies to any set $A \subset \mathbb{Z}$ with $|A+A|=3|A|+o(|A|)$ was accomplished by Jin [34]. His proof is a tour de force of non-standard analysis.

Lemma 2.6 (Jin). There exist an absolute positive constant $c$ and a natural number $K$ such that for every finite set $A$ of integers with $|A|>K$ and $|A+A|=3|A|-3+r$ for some integer $r$ with $0 \leq r \leq c|A|$, A satisfies at least one of the following properties:

(i) $A$ is a subset of an arithmetic progression of length $2|A|-1+2 r$;

(ii) $A \subseteq P_{1} \cup P_{2}$ for some arithmetic progressions $P_{1}, P_{2}$ with common step and $\left|P_{1}\right|+\left|P_{2}\right| \leq|A|+r$.

2.3. Large range. Here we give the proof of Lemma 2.1. We shall need a simple but crucial observation from [15, Proposition 2.1]. Its proof can be found in the appendix.

Lemma 2.7. Let $A$ be a sum-free set of positive integers and let $m$ be an arbitrary element of $A$. Then A satisfies the following conditions:

(i) $|A \cap([u, v] \cup[u+m, v+m])| \leq v-u+1$ for all $u, v \in \mathbb{N}$ with $u \leq v$;

(ii) $|A \cap[u, u+2 m-1]| \leq m$ for every $u \in \mathbb{N}$;

(iii) $|A \cap[u, v]| \leq \frac{1}{2}(v-u+m+1)$ for all $u, v \in \mathbb{N}$ with $u \leq v$.

We emphasise that in the first condition, the two intervals $[u, v]$ and $[u+m, v+m]$ are not necessarily disjoint.

We are now in position to prove Lemma 2.1.

Proof of Lemma 2.1. Throughout the proof let $m$ denote the minimum element of $A$. In the first step, we show that $m$ is not much larger than $n / 5$.

Claim 2.8. $m \leq(1 / 5+15 \eta) n$.

Proof. Suppose to the contrary that $m>(1 / 5+15 \eta) n$. As $m \in A$, we may apply Lemma 2.7 (ii) to $u=n-2 m+1$ and obtain

$$
|A \cap(n-2 m, n]| \leq m .
$$

Since $|A|>m$ by the assumption, this gives $m=\min (A) \leq n-2 m$, and so $m \leq n / 3$. One thus has

$$
(1 / 5+15 \eta) n \leq m \leq n / 3 .
$$

It follows from (2.3) that $[m, n]$ is covered by the intervals $\left[m, \frac{1}{2}(n-m)\right],\left(\frac{1}{2}(n-m), \frac{1}{2} n\right]$, $(n-2 m, n]$ and $\left[m+m, m+\frac{1}{2}(n-m)\right]$; so also is $A$ For the remainder of the proof we shall use this information to bound $|A|$.

Applying Lemma 2.7 (i) with $u=m$ and $v=\frac{1}{2}(n-m)$ we find

$$
\left|A \cap\left(\left[m, \frac{1}{2}(n-m)\right] \cup\left[m+m, m+\frac{1}{2}(n-m)\right]\right)\right| \leq n / 2-3 m / 2+1 .
$$

\footnotetext{
${ }^{2}$ One may verify this claim for $n / 5 \leq m \leq n / 4$, and for $n / 4 \leq m \leq n / 3$ separately.
} 
We next bound $\left|A \cap\left(\frac{1}{2}(n-m), \frac{1}{2} n\right]\right|$. For abbreviation, let $B=A \cap\left(\frac{1}{2}(n-m), \frac{1}{2} n\right]$. Using (2.1) gives

$$
|B| \leq|2 B| / 2+1 / 2 .
$$

To estimate $|2 B|$, we first observe that $2 B \subseteq[n-m+1, n]$ as $B \subseteq\left(\frac{1}{2}(n-m), \frac{1}{2} n\right]$, and $(A-A)_{+} \subseteq[n-m]$ since $A \subseteq[m, n]$. Moreover since $A$ is sum-free and $B \subseteq A$, we must have $A \cap 2 B=\emptyset$ and $A \cap(A-A)_{+}=\emptyset$. Hence $2 B,(A-A)_{+}$and $A$ are disjoint subsets of $[n]$, resulting in

$$
|2 B| \leq n-|A|-\left|(A-A)_{+}\right| .
$$

Note that $d(A)=1$ by the assumption, and $n-m \leq 2|A|-3$ by (2.3) and the assumption that $|A| \geq(2 / 5-\eta) n$ and $\eta \geq 1 / n$. Lemma 2.5 then implies

$$
|A|+\left|(A-A)_{+}\right| \geq \min \left\{\frac{3}{2}|A|+\frac{1}{2}(n-m-1), \frac{5}{2}|A|-2\right\}=\frac{3}{2}|A|+\frac{1}{2}(n-m-1) .
$$

Assembling all the information, we get

$$
\left|A \cap\left(\frac{1}{2}(n-m), \frac{1}{2} n\right]\right| \leq n / 4+m / 4-3|A| / 4+3 / 4 .
$$

Recalling that $A$ is covered by the intervals $\left[m, \frac{1}{2}(n-m)\right],\left(\frac{1}{2}(n-m), \frac{1}{2} n\right],(n-2 m, n]$ and $\left[m+m, m+\frac{1}{2}(n-m)\right]$, and using estimates (2.2), (2.4) and (2.5), we deduce that $|A| \leq 3 n / 4-m / 4-3|A| / 4+7 / 4$. Since $|A| \geq(2 / 5-\eta) n$ and $\eta \geq 1 / n$ by the assumption, this leads to $m \leq 3 n-7|A|+7 \leq(1 / 5+14 \eta) n$, which contradicts our hypothesis that $m \geq(1 / 5+15 \eta) n$.

In the second step, we establish an approximate version of the lemma.

Claim 2.9. All integers in $\left[\left(\frac{1}{5}+\sqrt{\eta}\right) n,\left(\frac{2}{5}-\sqrt{\eta}\right) n\right] \cup\left[\left(\frac{4}{5}+\sqrt{\eta}\right) n, n\right]$, with at most $14 \sqrt{\eta} n$ exceptions, belong to A.

Before proving Claim 2.9, we shall use it to finish the proof of Lemma 2.1. Suppose to the contrary that $A \nsubseteq\left[\left(\frac{1}{5}-\sqrt{\eta}\right) n,\left(\frac{2}{5}+32 \sqrt{\eta}\right) n\right] \cup\left[\left(\frac{4}{5}-31 \sqrt{\eta}\right) n, n\right]$. Then there exists $a \in A \cap\left[\left(\frac{2}{5}+32 \sqrt{\eta}\right) n,\left(\frac{4}{5}-31 \sqrt{\eta}\right) n\right]$ since $\min (A) \geq(1 / 5-\sqrt{\eta}) n$ by the assumption. From this we get

$$
a+(1 / 5+\sqrt{\eta}) n \leq(1-30 \sqrt{\eta}) n, \text { and } a+(2 / 5-\sqrt{\eta}) n \geq(4 / 5+31 \sqrt{\eta}) n,
$$

showing that the intervals $a+\left[\left(\frac{1}{5}+\sqrt{\eta}\right) n,\left(\frac{2}{5}-\sqrt{\eta}\right) n\right]$ and $\left[\left(\frac{4}{5}+\sqrt{\eta}\right) n, n\right]$ have at least $\min \{29 \sqrt{\eta} n,(1 / 5-3 \sqrt{\eta}) n\}=29 \sqrt{\eta} n$ elements in common. Thus, using pigeonhole principle and Claim 2.9, we find $a+b=c$ for some $b, c \in A$, which contradicts the assumption that $A$ is sum-free.

Finally we give a proof of Claim 2.9 using Claim 2.8, Lemmas 2.5 and 2.7.

Proof of Claim 2.9. As $(1 / 5-\sqrt{\eta}) n \leq m \leq(1 / 5+15 \eta) n$ and $\eta \leq 1 / 160^{2}$ by Claim 2.8 and the assumption, we have the following chain of inequalities:

$$
m \leq \frac{1}{2}(n-m) \leq \frac{1}{2} n \leq n-2 m \leq n-m \leq n .
$$

We shall use (2.6) to prove the claim which, roughly speaking, states that

$$
A \approx\left[m, \frac{1}{2}(n-m)\right] \cup(n-m, n] .
$$

(Note that $m \approx \frac{1}{5} n, \frac{1}{2}(n-m) \approx \frac{2}{5} n, n-2 m \approx \frac{3}{5} n$ and $n-m \approx \frac{4}{5} n$.)

Since $d(A)=1$, it follows from Lemma 2.5 that

$$
|A|+\left|(A-A)_{+}\right| \geq \min \left\{\frac{3}{2}|A|+\frac{1}{2}(n-m-1), \frac{5}{2}|A|-2\right\} \geq(1-10 \eta) n,
$$


where the last inequality holds since $m \leq(1 / 5+15 \eta) n$ by Claim 2.8 , and $|A| \geq(2 / 5-\eta) n$ and $\eta \geq 1 / n$ by the assumption. Moreover, as $A$ is a sum-free subset of $[m, n], A \cap[n-m]$ and $(A-A)_{+}$are disjoint subsets of $[n-m]$. Hence

$$
|A \cap(n-m, n]| \geq|A|+\left|(A-A)_{+}\right|-|[n-m]| \geq m-10 \eta n .
$$

Since $A$ is sum-free, $(2 \cdot A) \cap(n-m, n]$ and $A \cap(n-m, n]$ are disjoint, which gives

$$
\left|A \cap\left(\frac{1}{2}(n-m), \frac{1}{2} n\right]\right|=|(2 \cdot A) \cap(n-m, n]| \leq|(n-m, n] \backslash A| \stackrel{(2.7)}{\leq} 10 \eta n .
$$

We know from (2.6) that $\left|A \cap\left(\left[m, \frac{1}{2}(n-m)\right] \cup\left(\frac{1}{2} n, n-2 m\right]\right)\right|$ is at least

$$
|A|-\left|A \cap\left(\frac{1}{2}(n-m), \frac{1}{2} n\right]\right|-|A \cap(n-2 m, n]| \geq(2 / 5-11 \eta) n-m
$$

as $|A| \geq(2 / 5-\eta) n$ by the assumption, $\left|A \cap\left(\frac{1}{2}(n-m), \frac{1}{2} n\right]\right| \leq 10 \eta n$ by the previous estimate, and $|A \cap(n-2 m, n]| \leq m$ by Lemma 2.7 (ii).

We next apply Lemma 2.7 (i) with $u=\frac{1}{2} n-m$ and $v=n-3 m$ to obtain

$$
\left|A \cap\left(\left(\frac{1}{2} n-m, n-3 m\right] \cup\left(\frac{1}{2} n, n-2 m\right]\right)\right| \leq n / 2-2 m+1 .
$$

Using (2.6) once again, we may bound $\left|A \cap\left[m, \frac{1}{2} n-m\right]\right|$ from below by

$$
\begin{gathered}
\left|A \cap\left(\left[m, \frac{1}{2}(n-m)\right] \cup\left(\frac{1}{2} n, n-2 m\right]\right)\right|-\left|A \cap\left(\left(\frac{1}{2} n-m, n-3 m\right] \cup\left(\frac{1}{2} n, n-2 m\right]\right)\right| \\
\text { (by (2.8) and (2.9) }) \leq-(1 / 10+11 \eta) n+m-1 .
\end{gathered}
$$

This implies $|2 A \cap[2 m, n-2 m]| \geq-(1 / 5+22 \eta) n+2 m-3$, due to (2.1). Moreover, since $m \geq(1 / 5-\sqrt{\eta}) n$ and $\eta \leq 1 / 160^{2}$ by the assumption, one has $\left(\frac{1}{2} n, n-2 m\right] \subseteq[2 m, n-$ $2 m]$. We thus get $\left|\left(\frac{1}{2} n, n-2 m\right] \backslash 2 A\right| \leq|[2 m, n-2 m] \backslash 2 A| \leq(6 / 5+22 \eta) n-6 m+4$. From this and the assumption that $2 A \cap A=\emptyset$, we obtain

$$
\left|A \cap\left(\frac{1}{2} n, n-2 m\right]\right| \leq\left|\left(\frac{1}{2} n, n-2 m\right] \backslash 2 A\right| \leq(6 / 5+22 \eta) n-6 m+4
$$

Clearly we can bound $\left|\left[m, \frac{1}{2}(n-m)\right] \backslash A\right|$ from above by

$$
\left|\left[m, \frac{1}{2}(n-m)\right]\right|+\left|A \cap\left(\frac{1}{2} n, n-2 m\right]\right|-\left|A \cap\left(\left[m, \frac{1}{2}(n-m)\right] \cup\left(\frac{1}{2} n, n-2 m\right]\right)\right|
$$$$
\text { (by (2.10) and (2.8) }) \leq(13 / 10+33 \eta) n-13 m / 2+5 \leq 13 \sqrt{\eta} n
$$

assuming $m \geq(1 / 5-\sqrt{\eta}) n$ and $1 / n \leq \eta \leq 1 / 160^{2}$.

From (2.11) and (2.7) we see that all elements of $\left[m, \frac{1}{2}(n-m)\right] \cup(n-m, n]$ belong to $A$, with $(13 \sqrt{\eta}+10 \eta) n \leq 14 \sqrt{\eta} n$ exceptions. As $\left[m, \frac{1}{2}(n-m)\right] \cup(n-m, n]$ contains $\left[\left(\frac{1}{5}+\sqrt{\eta}\right) n,\left(\frac{2}{5}-\sqrt{\eta}\right) n\right] \cup\left[\left(\frac{4}{5}+\sqrt{\eta}\right) n, n\right]$ when $(1 / 5-\sqrt{\eta}) n \leq m \leq(1 / 5+15 \eta) n$ and $\eta \leq 1 / 160^{2}$, the claim follows.

2.4. Middle range. Our goal is to prove Lemma 2.2. For this purpose, we shall require the following variant of a fairly simple result due to Deshouillers et al. [15, Lemma 2.3]. We provide the proof in the appendix for completeness of exposition.

Lemma 2.10. Let $k \in \mathbb{N}$ and $\epsilon \geq 0$, and let $A \subseteq[0, k-1]$ and

$$
B=\left\{b_{1}<\ldots<b_{\ell}\right\}
$$

be two sets of integers such that $|A| \geq(1-\epsilon) k$ and $b_{i+1}-b_{i} \leq k$ for each $i \in[\ell-1]$. Then

$$
|A+B| \geq(1-4 \epsilon)(k+\ell(B)) .
$$

We are now able to prove Lemma 2.2 . 
Proof of Lemma 2.2. Throughout the proof let $m$ denote the minimum element of $A$. Suppose to the contrary that $|A| \geq(2 / 5-2 \eta) n$. Since $A$ is a sum-free subset of $[n]$, we thus have

$$
\left|(A-A)_{+}\right| \leq n-|A| \leq(3 / 5+2 \eta) n .
$$

To get a contradiction we seek to show $\left|(A-A)_{+}\right| \geq(3 / 5+3 \eta) n$. The following claim serves as an intermediate step.

Claim 2.11. Let $\epsilon=(5 \eta n+2) / m$, then we have

(i) $|A \cap[n-m+1, n]| \geq(1-\epsilon) m$,

(ii) $\left|(A-A)_{+} \cap[m-1]\right| \geq(1-\epsilon) m-1$.

Proof. (i) As $d(A)=1$ and $\ell(A)=n-m+1$, Lemma 2.5 gives

$$
|A|+\left|(A-A)_{+}\right| \geq \min \left\{\frac{3}{2}|A|+\frac{1}{2}(n-m-1), \frac{5}{2}|A|-2\right\} \geq(1-5 \eta) n-2
$$

for $m \leq n / 5$ and $|A| \geq(2 / 5-2 \eta) n$. Moreover $A \cap[n-m]$ and $(A-A)_{+}$are disjoint subsets of $[n-m]$ since $A$ is sum-free set in $[m, n]$. Therefore, we have

$$
|A \cap[n-m+1, n]| \geq|A|+\left|(A-A)_{+}\right|-|[n-m]| \geq m-(5 \eta n+2)=(1-\epsilon) m .
$$

(ii) It follows from (i) that

$$
\left|(A-A)_{+} \cap[m-1]\right| \geq|A \cap[n-m+1, n]|-1 \geq(1-\epsilon) m-1 .
$$

In the final step, we shall bound $\left|(A-A)_{+}\right|$from below.

Claim 2.12. $\left|(A-A)_{+}\right| \geq(3 / 5+3 \eta) n$.

Proof. Let $\left\{a_{1}<a_{2}<\ldots<a_{k}\right\}$ be the set consisting of all elements $a \in A \cap[n-2 m]$ such that $A \cap[a-m+1, a-1]=\emptyset$. Denote $a_{k+1}=n-2 m+1$, and $A_{i}=A \cap\left[a_{i}, a_{i+1}\right)$ for $i \in[k]$. It is not difficult to see that the following holds:

(*) For each $i \in[k]$, the gap between any two consecutive elements of $A_{i}$ is less than $m$.

Let $D=A \cap[n-m+1, n]$, and set $\epsilon=(5 \eta n+2) / m$. From Claim 2.11 (i) we have $|D| \geq(1-\epsilon) m$. Moreover, property $(*)$ implies that we may apply Lemma 2.10 to $A=D-(n-m+1)$ and $B=-A_{i}$, obtaining

$$
\left|D-A_{i}\right| \geq(1-4 \epsilon)\left(m+\ell\left(A_{i}\right)\right) \quad \text { for every } i \in[k] .
$$

Moreover, using parts (ii) and (iii) of Lemma 2.7 yields

$$
|A|=|A \cap[n-2 m+1, n]|+\sum\left|A_{i}\right| \leq m+\frac{1}{2} \sum\left(m+\ell\left(A_{i}\right)\right) .
$$

Furthermore, we can infer from property $(*)$ that $(A-A)_{+} \cap[m-1], D-A_{1}, \ldots, D-A_{k}$ are disjoint subsets of $(A-A)_{+}$. So

$$
\left|(A-A)_{+}\right| \geq\left|(A-A)_{+} \cap[m-1]\right|+\sum\left|D-A_{i}\right|
$$

(by Claim 2.11 (ii), (2.13) $) \geq(1-\epsilon) m-1+(1-4 \epsilon) \sum\left(m+\ell\left(A_{i}\right)\right)$

$$
\begin{aligned}
(\text { by }(2.14)) & \geq(1-\epsilon) m-1+(1-4 \epsilon)(2|A|-2 m) \\
& =(-1+7 \epsilon) m+(2-8 \epsilon)|A|-1 .
\end{aligned}
$$

Observe that $\epsilon=(5 \eta n+2) / m \leq \min \left\{\frac{1}{5} \sqrt{\eta}, \frac{1}{6}\right\}$ since $35 \sqrt{\eta} n \leq m$ and $1 / n \leq \eta$ by the assumption. Combining this with the assumption that $m \leq(1 / 5-\sqrt{\eta}) n$ and $|A| \geq(2 / 5-2 \eta) n$, we conclude that the right hand side of (2.15) is greater than

$$
(-1 / 5+\sqrt{\eta}) n+\left(4 / 5-\frac{4}{5} \sqrt{\eta}\right) n-1 \geq(3 / 5+3 \eta) n
$$


when $\eta \leq 1 / 175^{2}$. Hence $\left|(A-A)_{+}\right| \geq(3 / 5+3 \eta) n$, as promised.

Claim 2.12 obviously contradicts (2.12). This finishes our proof of Lemma 2.2.

2.5. Small range. This section is devoted to the proof of Lemma 2.3. As the proof is quite complicated, we first give a high level overview of our approach. Let $A_{0}=A \cap[n / 2]$. The proof naturally splits into four steps

1. Show that $\left|A_{0}\right| \geq(1 / 5-o(1)) n$ using Lemma 2.13 (i). This step is performed in Claim 2.14.

2. Use the estimate from the first step together with inverse theorems (Lemmas 2.4 and 2.6) to show that $A_{0} \subseteq I_{a} \cup I_{b}$, where $I_{a}=\left\{a, a+d, \ldots, a+\left(\ell_{a}-1\right) d\right\}$, $I_{b}=\left\{b, b+d, \ldots, b+\left(\ell_{b}-1\right) d\right\}$, and $\ell_{a}+\ell_{b}=(1+o(1))\left|A_{0}\right|$. This is performed in Claim 2.15,

3. Show that $A_{0}$ is contained in either $F_{1,4}$ or $F_{2,3}$ (Claim 2.16). This step is performed as follows:

3.1 Combining steps 1 and 2 and the property that $A_{0}$ is sum-free, we obtain a number of inequalities that must be satisfied by the endpoints of $I_{a}$ and $I_{b}$.

3.2 Use the inequalities from the previous step to show that $d=5$, and either $\{a, b\} \equiv\{1,4\}(\bmod 5)$ or $\{a, b\} \equiv\{2,3\}(\bmod 5)$.

4. We use a 'bootstrapping' argument (Lemma 2.13) to upgrade the '50\%-structured characterisation' of $A$ from step 3 to a 100\%-structured characterisation.

Our bootstrapping lemma is the following simple result, proven in the appendix, which states that if a set $A$ of integers is dense in some interval $I$, then the difference set and sumset of $A$ contain long subintervals of $I-I$ and $I+I$ respectively.

Lemma 2.13 (Folklore). Every finite set A of integers has the following properties:

(i) $A-A$ contains $[2|A|-\ell(A)-1]$;

(ii) If $A \subseteq[0, k]$ for some positive $k$, then $2 A$ contains $[2 k-2|A|+2,2|A|-2]$.

These properties are only useful when the size of $A$ is at least $\ell(A) / 2+1$, though it is convenient not to make this a requirement.

Proof of Lemma [2.3. Throughout the proof, let $A_{0}=A \cap[n / 2], A_{1}=A \backslash A_{0}$, and $m_{e}=\min (A \cap E)$. We shall use Lemma 2.13 to show that $\left|A_{0}\right|$ is relatively large.

Claim 2.14. $\left|A_{0}\right| \geq(1 / 5-38 \sqrt{\eta}) n$.

Proof. To obtain a contradiction, suppose $\left|A_{0}\right|<(1 / 5-38 \sqrt{\eta}) n$. As $|A| \geq(2 / 5-\eta) n$ by the assumption, this implies $\left|A_{1}\right|>(1 / 5+38 \sqrt{\eta}-\eta) n$. We shall divide the proof into two cases, depending on whether $d\left(A_{1}\right)=1$ or $d\left(A_{1}\right)>1$.

Case 1: $d\left(A_{1}\right)>1$.

We must have $d\left(A_{1}\right) \leq 2$, since otherwise $\left|A_{1}\right| \leq n / 6+1<(1 / 5+38 \sqrt{\eta}-\eta) n$, a contradiction. Thus $d\left(A_{1}\right)=2$, that is, either $A_{1} \subseteq E$ or $A_{1} \subseteq O$. In either case, Lemma 2.13 (i) shows that $A_{1}-A_{1}$ contains all the even numbers between 0 and $4\left|A_{1}\right|-n / 2 \geq 3 n / 10$, giving $m_{e} \geq 3 n / 10$.

We first consider the case $A_{1} \subseteq E$. As $m_{e} \geq 3 n / 10$, we have $\left|A_{0} \cap[n / 4]\right| \leq n / 8$, giving $\left|A_{0} \cap(n / 4, n / 2]\right| \geq\left|A_{0}\right|-n / 8$. Thus $2 \cdot A_{0} \cap(n / 2, n]$ contains at least $\left|A_{0}\right|-n / 8$ even numbers in $(n / 2, n] \backslash A_{1}$. (Note that $2 \cdot A_{0} \cap A_{1}=\emptyset$ since $A$ is sum-free.) It follows that the number of even integers in $(n / 2, n]$ is at least

$$
\left|A_{1}\right|+\left|A_{0}\right|-n / 8 \geq(2 / 5-\eta) n-n / 8>21 n / 80
$$


for $\eta$ small, which is impossible.

We are left with the case $A_{1} \subseteq O$. Let $M_{e}=\max (A \cap E)$, and let $O^{\prime}$ denote the set of all the odd numbers less than $M_{e}$ in $A$. We have already shown that $m_{e} \geq 3 n / 10$. In addition, since $A_{1} \subseteq O$, we have $n / 2 \geq M_{e}$. As $A$ is sum-free, $O^{\prime}+\left\{M_{e}\right\}$ is a subset of $\left\{M_{e}+1, M_{e}+3, \ldots, 2 M_{e}-1\right\} \backslash A$, and so $A$ has at most $M_{e} / 2-\left|O^{\prime}\right|$ odd elements in $\left[M_{e}, 2 M_{e}\right]$. Moreover, $\left(2 M_{e}, n\right]$ contains at most $\left(n-2 M_{e}\right) / 2$ odd numbers. Thus $|A \cap O| \leq\left|O^{\prime}\right|+\left(M_{e} / 2-\left|O^{\prime}\right|\right)+\left(n-2 M_{e}\right) / 2=\left(n-M_{e}\right) / 2$, and hence $|A \cap E|=|A|-|A \cap O| \geq(2 / 5-\eta) n-\left(n-M_{e}\right) / 2 \geq M_{e} / 2-n / 8$ when $\eta$ is small enough. However, $|A \cap E| \leq\left(M_{e}-m_{e}\right) / 2+1 \leq M_{e} / 2-3 n / 20+1$ since $m_{e} \geq 3 n / 20$. Using these bounds yields $3 n / 20-1 \leq n / 8$, which is impossible for $n$ large.

Case 2: $d\left(A_{1}\right)=1$.

Due to Lemma 2.13 (i), we have $A_{1}-A_{1} \supseteq\left[2\left|A_{1}\right|-\ell\left(A_{1}\right)-1\right]$. As $A$ is sum-free, it follows that $2\left|A_{1}\right|-\ell\left(A_{1}\right)-1<\min (A) \leq 35 \sqrt{\eta} n$, giving $\ell\left(A_{1}\right) \geq 2\left|A_{1}\right|-35 \sqrt{\eta} n-1$. Since $d\left(A_{1}\right)=1$, Lemma 2.5 implies

$$
\left|\left(A_{1}-A_{1}\right)_{+}\right| \geq \min \left\{\frac{1}{2}\left(\left|A_{1}\right|+\ell\left(A_{1}\right)-2\right), \frac{3}{2}\left|A_{1}\right|-2\right\} \geq \frac{3}{2}\left|A_{1}\right|-18 \sqrt{\eta} n
$$

for $1 / n \leq \eta \leq c$. Moreover, since $A_{0}$ and $\left(A_{1}-A_{1}\right)_{+}$are disjoint subsets of $[n / 2]$, we see that $n / 2 \geq\left|A_{0}\right|+\left|\left(A_{1}-A_{1}\right)_{+}\right|$. From these estimates we obtain

$$
n / 2 \geq\left|A_{0}\right|+3\left|A_{1}\right| / 2-18 \sqrt{\eta} n \geq(2 / 5-\eta) n+\left|A_{1}\right| / 2-18 \sqrt{\eta} n .
$$

So $\left|A_{1}\right| \leq(1 / 5+2 \eta+36 \sqrt{\eta}) n<(1 / 5+38 \sqrt{\eta}-\eta) n$ for small $\eta$, a contradiction.

In the rest of the proof, we use the $\kappa$-notation for constants tending to zero as their parameters do so, that is, $\kappa(\eta) \rightarrow 0$ whenever $\eta \rightarrow 0$.

We shall infer from Claim 2.14 that $\left|A_{0}+A_{0}\right| /\left|A_{0}\right|$ is small, and then rely on the inverse theorems (Lemmas 2.4 and 2.6) to get detailed structural information on $A_{0}$.

Claim 2.15. The set $A_{0}$ has the following properties:

(i) $d\left(A_{0}\right)=1$;

(ii) $A_{0} \subseteq P_{1} \cup P_{2}$ for some arithmetic progressions $P_{1}$ and $P_{2}$ with the same step and $\left|P_{1}\right|+\left|P_{2}\right| \leq(1+\kappa(\eta))\left|A_{0}\right|$.

Proof. (i) Toward a contradiction, suppose $d\left(A_{0}\right)>1$. We must have $d\left(A_{0}\right)<3$ because $\left|A_{0}\right| \geq(1 / 5-\kappa(\eta)) n>n / 6+1$ by Claim 2.14. Hence $A_{0} \subseteq E$ or $A_{0} \subseteq O$. If $A_{0} \subseteq E$, then $\left\{a / 2: a \in A_{0}\right\}$ is not sum-free since it is a set in $[n / 4]$ of size $\left|A_{0}\right|>n / 8+1$, contradicting our assumption that $A_{0}$ is sum-free. Now suppose $A_{0} \subseteq O$. Then $m_{e}>n / 2$. To bound $|A|$, we partition $A=A^{\prime} \cup A^{\prime \prime}$, in which $A^{\prime}=A \cap\left[m_{e}-1\right]$ and $A^{\prime \prime}=A \cap\left[m_{e}, n\right]$. Since $m_{e}=\min (A \cap E)$ and $A$ is sum-free, $A^{\prime}$ and $m_{e}-A^{\prime}$ are disjoint sets of odd numbers in $\left[m_{e}-1\right]$, giving $\left|A^{\prime}\right| \leq m_{e} / 4$. To deal with $A^{\prime \prime}$, we note that $A^{\prime \prime}-A^{\prime \prime}$ contains $\left[2\left|A^{\prime \prime}\right|-\left(n-m_{e}\right)-2\right]$ by Lemma 2.13 (i). As $A$ is sum-free, it follows that $2\left|A^{\prime \prime}\right|-\left(n-m_{e}\right)-2 \leq \min (A)=\kappa(\eta) n$, resulting in $\left|A^{\prime \prime}\right| \leq\left(n-m_{e}\right) / 2+\kappa(\eta) n$. Therefore, we have $|A|=\left|A^{\prime}\right|+\left|A^{\prime \prime}\right| \leq n / 2-m_{e} / 4+18 \sqrt{\eta} n \leq(1 / 4+\kappa(\eta)) n$, contradicting the assumption that $|A| \geq(2 / 5-\eta) n$.

(ii) Since $A$ is sum-free, $2 A_{0}$ and $A$ are disjoint subsets of $[n]$. Hence

$$
\left|2 A_{0}\right| \leq n-|A| \leq(3 / 5+\eta) n \leq(3+\kappa(\eta))\left|A_{0}\right|
$$

as $|A| \geq(2 / 5-\eta) n$ by the assumption, and $\left|A_{0}\right| \geq(1 / 5-\kappa(\eta)) n$ due to Claim 2.14. By applying Lemma 2.4 when $\left|2 A_{0}\right| \leq 3\left|A_{0}\right|-4$ and Lemma 2.6 in the case $\left|2 A_{0}\right| \geq$ $3\left|A_{0}\right|-3$, we deduce that $A_{0}$ satisfies one of the following conditions: 
(a) $A_{0}$ is a subset of an arithmetic progression of length $(2+\kappa(\eta))\left|A_{0}\right|$;

(b) $A_{0} \subseteq P_{1} \cup P_{2}$ for some arithmetic progressions $P_{1}$ and $P_{2}$ with the same step and $\left|P_{1}\right|+\left|P_{2}\right| \leq(1+\kappa(\eta))\left|A_{0}\right|$.

To prove property (ii), it thus suffices to show that case (a) is impossible. In this case $A_{0}$ is located in an interval of length $(2+\kappa(\eta))\left|A_{0}\right|$, as $d\left(A_{0}\right)=1$ by property (i). Since $\min \left(A_{0}\right)=\kappa(\eta) n$ by the assumption and $\left|A_{0}\right| \geq(1 / 5-\kappa(\eta)) n$ by Claim 2.14, it follows that $\min \left(A_{0}\right)=\kappa(\eta)\left|A_{0}\right|$ and $A_{0} \subseteq\left[(2+\kappa(\eta))\left|A_{0}\right|\right]$. By Theorem [1.1, we thus have $A_{0} \subseteq O$, which contradicts property (i).

We shall use the previous claims to obtain the following characterisation of $A_{0}$.

Claim 2.16. Either $A_{0} \subseteq F_{1,4}$ or $A_{0} \subseteq F_{2,3}$.

Before we proceed with the proof of Claim 2.16, we show how it implies the lemma. From Claim 2.16 we have $A_{0} \subseteq F_{1,4}$ or $A_{0} \subseteq F_{2,3}$. We shall show that if $A_{0} \subseteq F_{1,4}$ then $A \subseteq F_{1,4}$. Conversely, suppose that there exists $a \in A \backslash F_{1,4}$. Since $a \not \equiv 1,4(\bmod 5)$, we can find $i, j \in\{1,4\}$ such that $a \equiv i+j(\bmod 5)$. Now Claim 2.14 tells us that $\left|A_{0}\right| \geq(1 / 5-\kappa(\eta)) n$. Let $X=A_{0} \cap(5 \cdot \mathbb{Z}+1)$ and $Y=A_{0} \cap(5 \cdot \mathbb{Z}+4)$. From Lemma 2.13 (ii), we learn that $2 X, X+Y$ and $2 Y$ contain all the elements in $[\kappa(\eta) n,(1-\kappa(\eta)) n]$ of $5 \cdot \mathbb{Z}+2,5 \cdot \mathbb{Z}$ and $5 \cdot \mathbb{Z}+3$, respectively. As $2 A \neq A$ and $|A| \geq(2 / 5-\eta) n$, this shows that $a \geq(1-\kappa(\eta)) n$, and that $A$ contains all but at most $k(\eta) n$ elements of $[n] \cap(\{1,4\}+5 \cdot \mathbb{Z})$. Hence both $A$ and $a-A$ contain all but at most $\kappa(\eta) n$ elements of $[n] \cap(5 \cdot \mathbb{Z}+j)$, and so $A \cap(a-A) \neq \emptyset$, contradicting the assumption that $A \cap(A-A)=\emptyset$. In much the same way, the condition $A_{0} \subseteq F_{2,3}$ would force $A \subseteq F_{2,3}$.

We close this section by deducing Claim 2.16 from Claims 2.14 and 2.15.

Proof of Claim 2.16. Finally we come to what is, in some sense, the trickiest part of our proof. Due to Claim 2.15 (ii), there exist two arithmetic progressions $I_{a}=\{a, a+$ $\left.d, \ldots, a+\left(\ell_{a}-1\right) d\right\}$ and $I_{b}=\left\{b, b+d, \ldots, b+\left(\ell_{b}-1\right) d\right\}$ in $[n / 2]$ such that $A_{0} \subseteq I_{a} \cup I_{b}$ and $\left|I_{a}\right|+\left|I_{b}\right| \leq(1+\kappa(\eta))\left|A_{0}\right|$. In particular,

$$
\left|A_{0} \cap I_{u}\right| \geq\left|I_{u}\right|-\kappa(\eta) n \text { for every } u \in\{a, b\} .
$$

Clearly $\left|A_{0}\right| \leq\left|I_{a}\right|+\left|I_{b}\right| \leq n / d+2$. Combined with the bound $\left|A_{0}\right| \geq(1 / 5-\kappa(\eta)) n$ from Claim 2.14 we get $d \leq 5$. We distinguish three cases $d=1, d=2$ and $d \in\{3,4,5\}$.

Case 1: $d=1$.

In this case both $I_{a}$ and $I_{b}$ are intervals. Without loss of generality we can assume that $\left|I_{a}\right| \geq\left|I_{b}\right|$. Since $A_{0} \subseteq I_{a} \cup I_{b}$, it follows that $\left|I_{a}\right| \geq\left|A_{0}\right| / 2$, and so from (2.16) and Lemma 2.13 (i) we have $A_{0}-A_{0} \supseteq\left(A_{0} \cap I_{a}\right)-\left(A_{0} \cap I_{a}\right) \supseteq\left[(1 / 2-\kappa(\eta))\left|A_{0}\right|\right]$. But $\min \left(A_{0}\right)=\kappa(\eta)\left|A_{0}\right|$ by the assumption, resulting in $A_{0} \cap\left(A_{0}-A_{0}\right) \neq \emptyset$, a contradiction.

Case 2: $d=2$.

Since $d\left(A_{0}\right)=1$ by Claim 2.15 (i), we must have $A_{0} \cap I_{a} \neq \emptyset, A_{0} \cap I_{b} \neq \emptyset$ and $a \neq b(\bmod 2)$. So we can assume that $a \equiv 0(\bmod 2)$ and $b \equiv 1(\bmod 2)$. For $u \in\{a, b\}$, denote by $m_{u}$ the smallest element of $A \cap I_{u}$. We have $\min \left\{m_{a}, m_{b}\right\}=\min (A)=\kappa(\eta) n$ by the assumption. We thus have $m_{a} \leq n / 30$, or $m_{a}>n / 30$ and $m_{b}=\kappa(\eta) n$.

We first deal with the case $m_{a} \leq n / 30$. We claim that $\left|I_{u}\right| \leq n / 20$ for all $u \in\{a, b\}$. If this is not true then $\left|I_{u}\right| \geq n / 20$ for some $u \in\{a, b\}$. From (2.16) and Lemma 2.13 (i), it follows that $\left(A_{0} \cap I_{u}\right)-\left(A_{0} \cap I_{u}\right)$ contains all the even numbers between 1 and $(1-\kappa(\eta)) n / 20$. Since $m_{a} \leq n / 30$, this leads to $m_{a} \in A_{0}-A_{0}$, a contradiction. We thus have $\left|A_{0}\right| \leq\left|I_{a}\right|+\left|I_{b}\right| \leq n / 10$, contradicting Claim 2.14. 
We now consider the case $m_{a}>n / 30$ and $m_{b}=\kappa(\eta) n$. For $u \in\{a, b\}$, let $M_{u}$ be the largest element of $I_{u}$. As $I_{a} \subseteq[n / 2]$, we have the constraint $C 1: M_{a} \leq n / 2$. We next show that $m_{a}$ and $M_{a}$ satisfy $C 2: M_{a} \leq 2 m_{a}+n / 20$. Indeed if $M_{a} \geq 2 m_{a}+n / 20$, then we can deduce from (2.16) that $A_{0} \cap I_{a}$ and $m_{a}+\left(A_{0} \cap I_{a}\right)$ would have at least $(1-\kappa(\eta)) n / 40$ even elements in common, which contradicts the assumption that $A$ is sum-free. We shall need one more constraint $C 3: m_{a} \geq(2-\kappa(\eta)) M_{b}$. Indeed as $A_{0} \cap I_{a}$ is a sum-free subset of even integers in $[n / 2]$, we find $\left|A_{0} \cap I_{a}\right| \leq n / 4+1$. Together with the estimate $\left|A_{0}\right| \geq(1 / 5-\kappa(\eta)) n$ from Claim 2.14, we see that $\left|A_{0} \cap I_{b}\right| \geq(1 / 20-\kappa(\eta)) n$. From (2.16) and Lemma 2.13 (ii), it follows that $2\left(A_{0} \cap I_{b}\right)$ contains all the even numbers of $\left[\kappa(\eta) n,(2-\kappa(\eta)) M_{b}\right]$. As $A_{0}$ is sum-free and $m_{a}>n / 30$, this implies $m_{a} \geq(2-\kappa(\eta)) M_{b}$, as claimed. Under the constraints $C 1, C 2$ and $C 3$, one has

$$
\begin{aligned}
\left|A_{0}\right|=\left|A_{0} \cap I_{a}\right|+\left|A_{0} \cap I_{b}\right| & \leq \frac{1}{2}\left(M_{a}-m_{a}+2\right)+\frac{1}{2}\left(M_{b}+1\right) \\
& =\frac{3}{8} M_{a}+\frac{1}{8}\left(M_{a}-2 m_{a}\right)+\frac{1}{4}\left(2 M_{b}-m_{a}\right)+\frac{3}{2} \leq\left(\frac{31}{160}+\kappa(\eta)\right) n,
\end{aligned}
$$

which contradicts the lower bound $\left|A_{0}\right| \geq(1 / 5-\kappa(\eta)) n$ from Claim 2.14.

Case 3: $d \in\{3,4,5\}$.

For each $u \in\{a, b\}$, let $\alpha_{u}$ and $\beta_{u}$ be two real numbers such that $\min \left(I_{u}\right)=\alpha_{u} n / 2$ and $\max \left(I_{u}\right)=\beta_{u} n / 2$. Set $\epsilon=1 / 1000$. We first show that for $\eta>0$ sufficiently small, the parameters $\alpha_{a}, \beta_{a}, \alpha_{b}$ and $\beta_{b}$ satisfy the following constraints:

(C1) $0 \leq \alpha_{u} \leq \beta_{u} \leq 1$ for each $u \in\{a, b\}$,

$(C 2)\left(\beta_{a}-\alpha_{a}\right)+\left(\beta_{b}-\alpha_{b}\right) \geq 2 d / 5-\epsilon$,

(C3) If $u, v, w \in\{a, b\}$ and $u+v \equiv w(\bmod d)$, then $\beta_{u}+\beta_{v} \leq \alpha_{w}+\epsilon$ or $\beta_{w} \leq \alpha_{u}+\alpha_{v}+\epsilon$.

Indeed, as $I_{a}$ and $I_{b}$ are subsets of $[n / 2]$ the first constraint follows. The second holds since $\left|I_{a}\right|+\left|I_{b}\right| \geq\left|A_{0}\right| \geq(1 / 5-\kappa(\eta)) n$ by Claim 2.14. For the third, note first that from $(C 1)$ and $(C 2)$ one has

$$
\min \left\{\beta_{a}-\alpha_{a}, \beta_{b}-\alpha_{b}\right\} \geq 2 d / 5-\epsilon-1 \geq 0.193
$$

From (2.16) and (2.17) we find $\min \left\{\left|A_{0} \cap I_{a}\right|,\left|A_{0} \cap I_{b}\right|\right\} \geq 0.19 n /(2 d)-\kappa(\eta) n>0.01 n$. In particular, $A_{0} \cap I_{u}$ and $A_{0} \cap I_{v}$ are non-empty, and so (2.1) implies

$$
\begin{aligned}
\left|\left(A_{0} \cap I_{u}\right)+\left(A_{0} \cap I_{v}\right)\right| & \geq\left|A_{0} \cap I_{u}\right|+\left|A_{0} \cap I_{v}\right|-1 \\
(\text { by (2.16) })) & \geq\left|I_{u}\right|+\left|I_{v}\right|-1-\kappa(\eta) n=\left|I_{u}+I_{v}\right|-\kappa(\eta) n,
\end{aligned}
$$

where the last equality holds because $I_{u}$ and $I_{v}$ are two arithmetic progressions with the same step. It follows that $\left(A_{0} \cap I_{u}\right)+\left(A_{0} \cap I_{v}\right)$ contains all but at most $\kappa(\eta) n$ elements of the arithmetic progression $\left\{x \mid x \in\left[\left(\alpha_{u}+\alpha_{v}\right) \frac{n}{2},\left(\beta_{u}+\beta_{v}\right) \frac{n}{2}\right], x \equiv u+v(\bmod d)\right\}$. On the other hand, (2.16) tells us that $A_{0} \cap I_{w}$ contains all but at most $\kappa(\eta) n$ members of the arithmetic progression $\left\{x \mid x \in\left[\alpha_{w} n / 2, \beta_{w} n / 2\right], x \equiv w \equiv u+v(\bmod d)\right\}$. Therefore we must have $\beta_{u}+\beta_{v} \leq \alpha_{w}+\epsilon$ or $\beta_{w} \leq \alpha_{u}+\alpha_{v}+\epsilon$, as otherwise $\left(A_{0} \cap I_{u}\right)+\left(A_{0} \cap I_{v}\right)$ and $A_{0} \cap I_{w}$ would have at least $\min \left\{\epsilon \cdot \frac{n}{2 d}-\kappa(\eta) n, 0.01 n\right\}>0$ elements in common, which contradicts the assumption that $A_{0}$ is sum-free.

In what follows we shall exploit the constraints $(C 1)-(C 3)$ to show that the set $\{a, b\}$ is sum-free modulo $d$. Note that since $d\left(A_{0}\right)=1$, we must have $a \neq b\left(\bmod d^{\prime}\right)$ for every divisor $d^{\prime}$ of $d$ with $d^{\prime}>1$. Due to symmetry between $a$ and $b$, we thus only need to take care of the following three cases.

\footnotetext{
${ }^{3}$ In contrast, one may have $\min \left\{\beta_{a}, \beta_{b}\right\}=o(1)$ when $d=2$. This subtle difference between the two cases $d=2$ and $d \geq 3$ has forced us to treat them separately.
} 
Case 3.1: $d=3, a \equiv 1(\bmod 3)$ and $b \equiv 2(\bmod 3)$. Using $(C 3)$ with $u=v=a$ and $w=b$, we deduce that $2 \beta_{a} \leq \alpha_{b}+\epsilon$ or $\beta_{b} \leq 2 \alpha_{a}+\epsilon$. If $2 \beta_{a} \leq \alpha_{b}+\epsilon$, then

$$
\left(\beta_{a}-\alpha_{a}\right)+\left(\beta_{b}-\alpha_{b}\right)=\beta_{b}+\left(2 \beta_{a}-\alpha_{b}\right)-\left(\beta_{a}+\alpha_{a}\right) \leq 1+\epsilon<2 d / 5-\epsilon
$$

since $\beta_{b} \leq 1$ and $\alpha_{a}, \beta_{a} \geq 0$ by $(C 1)$, which contradicts $(C 2)$. We thus have $\beta_{b} \leq 2 \alpha_{a}+\epsilon$. By symmetry we also get $\beta_{a} \leq 2 \alpha_{b}+\epsilon$. Hence

$$
\left(\beta_{a}-\alpha_{a}\right)+\left(\beta_{b}-\alpha_{b}\right)=\frac{1}{2}\left(\beta_{a}+\beta_{b}\right)+\frac{1}{2}\left(\beta_{a}-2 \alpha_{b}\right)+\frac{1}{2}\left(\beta_{b}-2 \alpha_{a}\right) \leq 1+\epsilon
$$

since $\beta_{a}, \beta_{b} \leq 1$ by $(C 1)$. But this bound is inconsistent with $(C 2)$.

Case 3.2: $a \equiv 0(\bmod d)$. Property $(C 3)$ tells us that $\beta_{a}+\beta_{b} \leq \alpha_{b}+\epsilon$ or $\beta_{b} \leq$ $\alpha_{a}+\alpha_{b}+\epsilon$. If the former condition occurs, then from $(C 1)$ and $(C 2)$ we get

$$
1+\epsilon \geq \alpha_{b}+\epsilon \geq \beta_{a}+\beta_{b} \geq\left(\beta_{a}-\alpha_{a}\right)+\left(\beta_{b}-\alpha_{b}\right) \geq 2 d / 5-\epsilon,
$$

which is impossible. Hence $\beta_{b} \leq \alpha_{a}+\alpha_{b}+\epsilon$. Combined with the constraint $\beta_{a} \leq 1$ from $(C 1)$, we again get a contradiction

$$
\left(\beta_{a}-\alpha_{a}\right)+\left(\beta_{b}-\alpha_{b}\right)=\beta_{a}+\left(\beta_{b}-\alpha_{a}-\alpha_{b}\right) \leq 1+\epsilon<2 d / 5-\epsilon .
$$

Case 3.3: $d \in\{4,5\}, a, b \not \equiv 0(\bmod d)$, and $a \not \equiv b\left(\bmod d^{\prime}\right)$ for every divisor $d^{\prime}$ of $d$ with $d^{\prime}>1$. We begin by reducing to the case that $\{a, b\}$ is sum-free modulo $d$. Indeed consider the relation $b \equiv 2 a(\bmod d)$. As in the proof of Case 3.1, this would imply $\beta_{b} \leq 2 \alpha_{a}+\epsilon$. Thus

$$
\left(\beta_{a}-\alpha_{a}\right)+\left(\beta_{b}-\alpha_{b}\right)=\left(\beta_{a}+\frac{1}{2} \beta_{b}\right)+\frac{1}{2}\left(\beta_{b}-2 \alpha_{a}\right)-\alpha_{b} \leq 3 / 2+\epsilon / 2,
$$

since $\beta_{a}, \beta_{b} \leq 1$ and $\alpha_{b} \geq 0$ by $(C 1)$. But once again this contradicts $(C 2)$. The case $a \equiv 2 b(\bmod d)$ follows by symmetry.

We have shown that the set $\{a, b\}$ is sum-free modulo $d$. Combined this with the condition that $a \not \equiv b\left(\bmod d^{\prime}\right)$ for every divisor $d^{\prime}$ of $d$ with $d^{\prime}>1$, we conclude that, up to a permutation of $a$ and $b$, either $a \equiv 1(\bmod 5)$ and $b \equiv 4(\bmod 5)$ or $a \equiv 2(\bmod 5)$ and $b \equiv 3(\bmod 5)$.

\section{THE NUMBER OF 2-WISE SUM-FREE SETS}

In this section, we prove Theorem 1.5 .

3.1. Proof overview. Recently the method of containers has emerged as a powerful tool for tackling various problems in combinatorics. Roughly speaking this method states that the independent sets in many 'natural' hypergraphs exhibit a certain kind of 'clustering', which allows one to count them one cluster at a time. Balogh, Morris and Samotij [10] and Saxton and Thomason [42], proved general container theorems for hypergraphs $\mathcal{H}$ whose edges are fairly 'evenly distributed' over the vertices of $\mathcal{H}$.

In the proof of Theorem 1.5, we shall apply a special case of a container result of Hancock, Staden and Treglown [30, Theorem 4.7]. We remark that their proof uses the theorems of Balogh, Morris and Samotij [10, and Saxton and Thomason [42].

Lemma 3.1 (Hancock-Staden-Treglown). There exists a collection $\mathcal{C}$ of subsets of $[n]^{2}$ with the following three properties:

(i) If $\left(A_{1}, A_{2}\right)$ is a pair of disjoint sum-free subsets of $[n]$, then there exists a pair $\left(C_{1}, C_{2}\right) \in \mathcal{C}$ such that $\left(A_{1}, A_{2}\right) \subseteq\left(C_{1}, C_{2}\right)$;

(ii) $|\mathcal{C}|=2^{o(n)}$;

(iii) For any $\left(C_{1}, C_{2}\right) \in \mathcal{C}$, each $C_{i}$ contains at most o $\left(n^{2}\right)$ Schur triples. 
We refer to the elements of $\mathcal{C}$ from Lemma 3.1 as containers.

A counting strategy. Our general strategy is influenced by the approach used in [9], which in turn dates back to earlier s of Cameron and Erdös [12] and Green [26]. Given $A \in \mathrm{SF}_{2}(n)$ and a partition $A=A_{1} \cup A_{2}$ of $A$ into two sum-free sets, we consider some container $\left(C_{1}, C_{2}\right) \in \mathcal{C}$ with $\left(A_{1}, A_{2}\right) \subseteq\left(C_{1}, C_{2}\right)$. As $\mathcal{C}$ is so small, the number of $A$ for which $\left|C_{1} \cup C_{2}\right| \leq(4 / 5-\eta) n$ is $o\left(2^{4 n / 5}\right)$. If, however, $\left|C_{1} \cup C_{2}\right| \geq(4 / 5-\eta) n$ then it is possible to say something about the structure of $\left(C_{1}, C_{2}\right)$, and hence about the structure of a typical set $A \in \mathrm{SF}_{2}(n)$. We then use a direct argument rather than counting such sets within the containers.

As discussed above, we need to get a handle on the structure of large containers. For this purpose, we first deduce from Theorem 1.4 a structural result on 2 -wise sum-free sets of size close to $4 n / 5$, which may be of independent interest.

Proposition 3.2. There exists an absolute positive constant $c$ such that the following holds for every $n \in \mathbb{N}$ and every $\eta \in \mathbb{R}$ with $2 / n \leq \eta \leq c$. Let $C_{1}$ and $C_{2}$ be two sum-free sets (not necessarily disjoint) in $[n]$ with $\left|C_{1} \cup C_{2}\right| \geq(4 / 5-\eta) n$. Then, up to a permutation of $C_{1}$ and $C_{2}$, one of the following situations occurs:

(i) $\left|C_{1} \backslash F_{1,4}\right|+\left|C_{2} \backslash F_{2,3}\right| \leq 14 \eta n$;

(ii) $\left|C_{1} \backslash I_{1}\right|+\left|C_{2} \backslash I_{2}\right| \leq 2424 \sqrt{\eta} n$, where $I_{1}=\left(\frac{n}{5}, \frac{2 n}{5}\right] \cup\left(\frac{4 n}{5}, n\right]$ and $I_{2}=\left(\frac{2 n}{5}, \frac{4 n}{5}\right]$.

Proof of Proposition 3.2. We begin by showing that neither $\left|C_{1}\right|$ nor $\left|C_{2}\right|$ are substantially greater than $2 n / 5$.

Claim 3.3. $\max \left\{\left|C_{1}\right|,\left|C_{2}\right|\right\} \leq(2 / 5+3 \eta) n$.

Proof. Denote $\tilde{C}_{2}=C_{2} \backslash C_{1}$ and $R=[n] \backslash\left(C_{1} \cup C_{2}\right)$. As $\left|C_{1} \cup C_{2}\right| \geq(4 / 5-\eta) n$, one has $|R| \leq(1 / 5+\eta) n$. Let $c_{1}, \ldots, c_{k}$ be the elements of $C_{1}$, indexed in increasing order, and let $D=\left\{c_{2}-c_{1}, \ldots, c_{k}-c_{1}\right\}$. Since $C_{1}$ is sum-free, $D \cap C_{1}=\emptyset$, and consequently $D \subseteq \tilde{C}_{2} \cup R$. It follows that

$$
\left|D \cap \tilde{C}_{2}\right| \geq|D|-|R|
$$

Let $\ell=\left|D \cap \tilde{C}_{2}\right|$. From the definition of $D$, there are $\ell$ distinct numbers $i_{1}, \ldots, i_{\ell}$ in $\{2,3, \ldots, k\}$, indexed in increasing order, so that $D \cap \tilde{C}_{2}=\left\{c_{i_{1}}-c_{1}, \ldots, c_{i_{\ell}}-c_{1}\right\}$. Since $\tilde{C}_{2}$ is sum-free, one has $c_{i_{t}}-c_{i_{1}}=\left(c_{i_{t}}-c_{1}\right)-\left(c_{i_{1}}-c_{1}\right) \notin \tilde{C}_{2}$ for all $t$ with $2 \leq t \leq \ell$. Also $c_{i_{t}}-c_{i_{1}} \notin C_{1}$, as $C_{1}$ is sum-free. Hence $c_{i_{t}}-c_{i_{1}} \in R$ for each $t \in\{2, \ldots, \ell\}$, and so $|R| \geq \ell-1$. Thus

$$
\left|D \cap \tilde{C}_{2}\right| \leq|R|+1
$$

Using these bounds on $\left|D \cap \tilde{C}_{2}\right|$ gives $\left|C_{1}\right|=|D|+1 \leq 2|R|+2 \leq(2 / 5+3 \eta) n$ when $\eta \geq 1 / n$. In the same manner we can show $\left|C_{2}\right| \leq(2 / 5+3 \eta) n$.

We consider the sets $\tilde{C}_{1}=C_{1} \backslash C_{2}$ and $\tilde{C}_{2}=C_{2} \backslash C_{1}$. Clearly one has $\tilde{C}_{1} \cap \tilde{C}_{2}=\emptyset$. Since $\max \left\{\left|C_{1}\right|,\left|C_{2}\right|\right\} \leq(2 / 5+3 \eta) n$ by Claim 3.3 and $\left|C_{1} \cup C_{2}\right| \geq(4 / 5-\eta) n$ by the assumption, we find $\left|C_{1} \cap C_{2}\right| \leq 7 \eta n$ and $\min \left\{\left|\tilde{C}_{1}\right|,\left|\tilde{C}_{2}\right|\right\} \geq(2 / 5-4 \eta) n$. We shall derive the lemma from this information and Theorem 1.4.

Applying Theorem 1.4 to $\tilde{C}_{1}$ and $\tilde{C}_{2}$, and noting that $\min \left\{\left|\tilde{C}_{1}\right|,\left|\tilde{C}_{2}\right|\right\} \geq(2 / 5-4 \eta) n$ and $\tilde{C}_{1} \cap \tilde{C}_{2}=\emptyset$, we conclude that, up to a permutation of $\tilde{C}_{1}$ and $\tilde{C}_{2}$, one of the following conditions must be true:

(i') $\tilde{C}_{1} \subseteq F_{1,4}$ and $\tilde{C}_{2} \subseteq F_{2,3}$; 
(ii') $\tilde{C}_{1} \subset \tilde{I}_{1}$ and $\min \left(\tilde{C}_{2}\right) \geq(2 / 5-4 \eta) n$, where

$$
\tilde{I}_{1}=\left[\left(\frac{1}{5}-400 \sqrt{\eta}\right) n,\left(\frac{2}{5}+400 \sqrt{\eta}\right) n\right] \cup\left[\left(\frac{4}{5}-400 \sqrt{\eta}\right) n, n\right] .
$$

If condition (i') holds, then $\left|C_{1} \backslash F_{1,4}\right|+\left|C_{2} \backslash F_{2,3}\right| \leq 2\left|C_{1} \cap C_{2}\right| \leq 14 \eta n$. Suppose, then, that condition (ii') is true. In particular, one has $\left|\tilde{C}_{1} \backslash I_{1}\right| \leq 1200 \sqrt{\eta} n+3$. Hence

$$
\left|C_{1} \backslash I_{1}\right| \leq\left|\tilde{C}_{1} \backslash I_{1}\right|+\left|C_{1} \cap C_{2}\right| \leq(1200 \sqrt{\eta}+7 \eta) n+3,
$$

as $\left|C_{1} \cap C_{2}\right| \leq 7 \eta n$.

It remains to bound $\left|C_{2} \backslash I_{2}\right|$. From condition (ii') and the fact that $\tilde{C}_{1} \cap \tilde{C}_{2}=\emptyset$, we learn that $\tilde{C}_{2} \backslash I_{2} \subseteq \tilde{C}_{2} \cap \tilde{I}_{1} \subseteq \tilde{I}_{1} \backslash \tilde{C}_{1}$. Thus $C_{2} \backslash I_{2} \subseteq\left(\tilde{C}_{2} \backslash I_{2}\right) \cup\left(C_{1} \cap C_{2}\right) \subseteq$ $\left(\tilde{I}_{1} \backslash \tilde{C}_{1}\right) \cup\left(C_{1} \cap C_{2}\right)$, leading to

$$
\left|C_{2} \backslash I_{2}\right| \leq\left|\tilde{I}_{1} \backslash \tilde{C}_{1}\right|+\left|C_{1} \cap C_{2}\right|=\left|\tilde{I}_{1}\right|-\left|\tilde{C}_{1}\right|+\left|C_{1} \cap C_{2}\right| \leq(1200 \sqrt{\eta}+11 \eta) n+3,
$$

where the second inequality follows from condition (ii'), and in the last we evaluated $\left|\tilde{I}_{1}\right| \leq(2 / 5+1200 \sqrt{\eta})+3,\left|\tilde{C}_{1}\right| \geq(2 / 5-4 \eta) n$ and $\left|C_{1} \cap C_{2}\right| \leq 7 \eta n$. From these upper bounds on $\left|C_{1} \backslash I_{1}\right|$ and $\left|C_{2} \backslash I_{2}\right|$, we find

$$
\left|C_{1} \backslash I_{1}\right|+\left|C_{2} \backslash I_{2}\right| \leq(2400 \sqrt{\eta}+18 \eta) n+6 \leq 2424 \sqrt{\eta} n .
$$

We also need a removal lemma of Green [27, Corollary 1.6] for sum-free sets.

Lemma 3.4 (Green). Suppose that $C \subseteq[n]$ is a set containing o $\left(n^{2}\right)$ Schur triples. Then there exists a sum-free subset $\tilde{C}$ of $C$ such that $|C \backslash \tilde{C}|=o(n)$.

From Lemma 3.1, Proposition 3.2 and Lemma 3.4 we obtain the following description of almost all $A \in \mathrm{SF}_{2}(n)$. Note that we shall identify each set $A \in \mathrm{SF}_{2}(n)$ with a pair $\left(A_{1}, A_{2}\right)$ of disjoint sum-free sets so that $A=A_{1} \cup A_{2}$.

Corollary 3.5. Given $\delta>0$, every set $A \in \mathrm{SF}_{2}(n)$, with at most o $\left(2^{4 n / 5}\right)$ exceptions, has one of the following structures (up to a permutation of $A_{1}$ and $A_{2}$ ):

(a) $\left|A_{1} \backslash F_{1,4}\right|+\left|A_{2} \backslash F_{2,3}\right| \leq \delta n$;

(b) $\left|A_{1} \backslash I_{1}\right|+\left|A_{2} \backslash I_{2}\right| \leq \delta n$, in which $I_{1}=\left(\frac{n}{5}, \frac{2 n}{5}\right] \cup\left(\frac{4 n}{5}, n\right]$ and $I_{2}=\left(\frac{2 n}{5}, \frac{4 n}{5}\right]$.

In the remainder of the paper we refer to sets that satisfy condition (a) and condition (b) from Corollary 3.5 as type $(a)$ and type (b) respectively. Note that Corollary 3.5 implies that, in order to prove Theorem 1.5, it suffices to show that there are at most $O\left(2^{4 n / 5}\right)$ sets $A \in \mathrm{SF}_{2}(n)$ of type (a) and type (b).

Proof of Corollary 3.5. Let $\eta=\min \left\{\frac{\delta}{29},\left(\frac{\delta}{3430}\right)^{2}, \frac{1}{2} q 3.2\right\}$, where 93.2 is the absolute positive constant from Proposition 3.2. For each set $A \in \mathrm{SF}_{2}(n)$, we fix a pair $\left(A_{1}, A_{2}\right)$ of disjoint sum-free sets in $[n]$, and a container $\left(C_{1}, C_{2}\right) \in \mathcal{C}$ such that $A=A_{1} \cup A_{2}$ and $\left(A_{1}, A_{2}\right) \subset\left(C_{1}, C_{2}\right)$. According to Lemma 3.1 (ii), the number of set $A \in \mathrm{SF}_{2}(n)$ for which $\left|C_{1} \cup C_{2}\right| \leq(4 / 5-\eta) n$ is certainly at most $2^{(4 / 5-\eta) n} \cdot 2^{o(n)}=o\left(2^{4 n / 5}\right)$, so suppose $\left|C_{1} \cup C_{2}\right| \geq(4 / 5-\eta) n$. By Lemma 3.4, there exists a pair $\left(\tilde{C}_{1}, \tilde{C}_{2}\right)$ of sumfree sets such that $\left(\tilde{C}_{1}, \tilde{C}_{2}\right) \subseteq\left(C_{1}, C_{2}\right)$ and $\left|C_{1} \backslash \tilde{C}_{1}\right|+\left|C_{2} \backslash \tilde{C}_{2}\right|=o(n)$. Observe that $\left|\tilde{C}_{1} \cup \tilde{C}_{2}\right|=\left|C_{1} \cup C_{2}\right|-o(n) \geq(4 / 5-2 \eta) n$. Since $2 \eta \leq q 3.2$ by the choice of $\eta$, we may appeal to Proposition 3.2 to conclude that:

$\left(a^{\prime}\right)\left|\tilde{C}_{1} \backslash F_{1,4}\right|+\left|\tilde{C}_{2} \backslash F_{2,3}\right| \leq 28 \eta n$, or

$\left(b^{\prime}\right)\left|\tilde{C}_{1} \backslash I_{1}\right|+\left|\tilde{C}_{2} \backslash I_{2}\right| \leq 3429 \sqrt{\eta} n$. 
If case $\left(a^{\prime}\right)$ is true, then $\left|A_{1} \backslash F_{1,4}\right|+\left|A_{2} \backslash F_{2,3}\right| \leq 28 \eta n+o(n)<\delta n$ for $\eta \leq \delta / 29$. If, however, case $\left(b^{\prime}\right)$ occurs then $\left|A_{1} \backslash I_{1}\right|+\left|A_{2} \backslash I_{2}\right| \leq 3429 \sqrt{\eta} n+o(n) \leq \delta n$ since $\eta \leq\left(\frac{\delta}{3430}\right)^{2}$, completing the proof.

3.2. Restricted partitions and sumsets. In this section, we introduce some tools that are useful for counting sets $A \in \mathrm{SF}_{2}(n)$ of type (a) and type (b).

A handy tool for the study of sumsets is Plünnecke's inequality [39].

Lemma 3.6 (Plünnecke Inequality). If $S$ is a set of integers and $|S+S| \leq R|S|$, then

$$
|k S| \leq R^{k}|S|
$$

for any positive integer $k$.

We shall need the following bound on the number of $s$-subsets $S$ of $\{1,2, \ldots, D\}$ with $|S+S| \leq R|S|$, due to Green and Morris [29, Theorem 1.1].

Lemma 3.7 (Green-Morris). Fix $\delta>0$ and $R>0$. Then the following holds for all integers $s$ with $s \geq s_{0}(\delta, R)$. For any $D \in \mathbb{N}$ there are at most

$$
2^{\delta s}\left(\begin{array}{c}
\frac{1}{2} R s \\
s
\end{array}\right) D^{\lfloor R+\delta\rfloor}
$$

sets $S \subseteq[D]$ with $|S|=s$ and $|S+S| \leq R|S|$.

Lemma 3.7 will be used in conjunction with some estimates on binomial coefficients, which we list here for future reference. It is well-known that for every integers $n$ and $k$ with $0 \leq k \leq n$ and every real $\alpha$ with $0 \leq \alpha \leq 1 / 2$, we have

$$
\left(\begin{array}{l}
n \\
k
\end{array}\right) \leq 2^{H(k / n) n} \text {, and } \sum_{i \leq \alpha n}\left(\begin{array}{l}
n \\
i
\end{array}\right) \leq 2^{H(\alpha) n},
$$

where $H(x)=-x \log _{2}(x)-(1-x) \log _{2}(1-x)$ is the binary entropy function.

Another component in our argument is a crude bound on the number of restricted integer partitions (see [4, Lemma 5.1]).

Lemma 3.8. Given $k, \ell \in \mathbb{N}$, let $p_{\ell}^{*}(k)$ denote the number of integer partitions of $k$ into $\ell$ distinct parts. Then

$$
p_{\ell}^{*}(k) \leq\left(\frac{e^{2} k}{\ell^{2}}\right)^{\ell}
$$

To handle sets with large sumset, we shall apply the following lower tail estimate, which is a special case of Janson's inequality (see [33, Theorem 2.14]).

Lemma 3.9 (Janson Inequality). Suppose that $\left\{U_{i}\right\}_{i \in I}$ is a collection of subsets of a finite set $\Gamma$. Let

$$
\mu=\sum_{i \in I}\left(\frac{1}{2}\right)^{\left|U_{i}\right|} \quad \text { and } \quad \Delta=\sum_{i \sim j}\left(\frac{1}{2}\right)^{\left|U_{i} \cup U_{j}\right|}
$$

where the second sum is over ordered pairs $(i, j)$ such that $i \neq j$ and $U_{i} \cap U_{j} \neq \emptyset$. Then the number of subsets of $\Gamma$ that contain at most $\mu / 2$ sets $U_{i}$ is at most

$$
e^{-\mu^{2} /(8 \mu+8 \Delta)} \cdot 2^{|\Gamma|}
$$


3.3. Counting sets of type $(a)$ and type $(b)$. Throughout we identify each set $A \in \mathrm{SF}_{2}(n)$ with a pair $\left(A_{1}, A_{2}\right)$ of disjoint sum-free sets so that $A=A_{1} \cup A_{2}$.

The following lemma deals with sets of type $(a)$.

Lemma 3.10. There are $(1+o(1)) 2^{\lceil 4 n / 5\rceil}$ sets $A \in \mathrm{SF}_{2}(n)$ of type $(a)$, provided that $\delta>0$ is sufficiently small.

Proof. There are $2^{\lceil 4 n / 5\rceil}$ sets $A \in \mathrm{SF}_{2}(n)$ with $A_{1} \subseteq F_{1,4}$ and $A_{2} \subseteq F_{2,3}$. So, to prove the lemma, it suffices to show that the number of type $(a)$ sets $A$ with $0<\left|A_{1} \backslash F_{1,4}\right|+$ $\left|A_{2} \backslash F_{2,3}\right| \leq \delta n$ is $o\left(2^{4 n / 5}\right)$. By symmetry we only need to deal with the case that $A_{1} \backslash F_{1,4}$ contains at least one element, $t$ say. If $t<n / 2$, then we may select $n / 20$ disjoint pairs $(x, x+t)$ in $F_{1,4}$, and $A_{1}$ can not contain both of the elements of any of them since it is sum-free. The number of choices for the pair $\left(A_{1} \cap F_{1,4}, A_{2} \cap F_{2,3}\right)$ is thus no more than $2^{3 n / 10} 3^{n / 20} \cdot 2^{2 n / 5}=2^{7 n / 10} 3^{n / 20}$. Furthermore, since $\left|A_{1} \backslash F_{1,4}\right|+\left|A_{2} \backslash F_{2,3}\right| \leq \delta n$, the number of pairs $\left(A_{1} \backslash F_{1,4}, A_{2} \backslash F_{2,3}\right)$ is at most $\left(\sum_{i<\delta n}\left(\begin{array}{c}n \\ i\end{array}\right)\right)^{2} \leq 2^{2 H(\delta) n}$, due to (3.1). We deduce that there are at most $2^{7 n / 10} 3^{n / 20} \cdot 2^{2 H(\delta) n}=o\left(2^{4 n / 5}\right)$ ways to choose $\left(A_{1}, A_{2}\right)$. If $t \geq n / 2$ then a very similar argument applies with pairs $(x, x-t)$.

We now turn our attention to sets of type $(b)$. Note that Corollary 3.5, Lemmas 3.10. and 3.11 together imply Theorem 1.5 .

Lemma 3.11. If $\delta>0$ is sufficiently small, then there are $O\left(2^{4 n / 5}\right)$ sets $A \in \mathrm{SF}_{2}(n)$ of type $(b)$.

The proof of Lemma 3.11 is fairly long and technical so, in order to aid the reader, we shall start by giving a brief sketch. The argument is split into four claims; the first three being relatively straightforward, and the last being somewhat more involved.

We begin, in Claim 3.12, by using a direct argument to give a description of almost all sets $A \in \mathrm{SF}_{2}(n)$ of type $(b)$. In Claims 3.13, 3.14 and 3.15, we use this description to bound the number of sets $A \in \mathrm{SF}_{2}(n)$ with $S=A \cap[n / 5]$ fixed. Specifically, writing $\ell=|S|$ and $k=\sum_{a \in S}(n / 5-a)$, in Claim 3.13 we use Claim 3.12, Lemmas 3.8 and 3.9 to deal with the case $k \gg \ell^{2}$. Then, in Claim 3.14, we use Claim 3.12 and Lemma 3.8 to handle the case $k=O\left(\ell^{2}\right)$ and $|S+S| \gg|S|$. Finally, in Claim 3.15, we treat the remaining (hard) case; however, since we now have $|S+S|=O(|S|)$, we may apply Lemma 3.7 in place of Lemma 3.8 .

Proof of Lemma 3.11. Fix $\delta>0$ sufficiently small, and let $n \in \mathbb{N}$. We shall show that there are at most $O\left(2^{4 n / 5}\right)$ sets $A \in \mathrm{SF}_{2}(n)$ of type $(b)$. Since for us the residue of $n$ modulo 5 will not matter, we assume for simplicity throughout the proof that $n$ is divisible by 5 . We begin by proving that a typical set $A \in \mathrm{SF}_{2}(n)$ of type $(b)$ has the following property:

$(\alpha) A_{1} \subseteq\left[\left(\frac{1}{5}-\frac{1}{100}\right) n,\left(\frac{2}{5}+\frac{1}{100}\right) n\right] \cup\left[\left(\frac{4}{5}-\frac{1}{100}\right) n, n\right]$ and $A_{2} \subseteq\left[\left(\frac{2}{5}-\frac{1}{100}\right) n,\left(\frac{4}{5}+\frac{1}{100}\right) n\right]$.

Claim 3.12. With $o\left(2^{4 n / 5}\right)$ exceptions, all sets $A \in \mathrm{SF}_{2}(n)$ of type (b) satisfy $(\alpha)$.

Proof. Let $A \in \mathrm{SF}_{2}(n)$ be a set of type (b) that does not posses property $(\alpha)$. If $A_{1}$ contains an element $t \in\left[\left(\frac{1}{5}-\frac{1}{100}\right) n\right] \cup\left[\left(\frac{2}{5}+\frac{1}{100}\right) n,\left(\frac{4}{5}-\frac{1}{100}\right) n\right]$, then we can pick at least $n / 400$ disjoint pairs $(x, x+t)$ in $I_{1}$. Thus the number of ways to choose $\left(A_{1} \cap I_{1}, A_{2} \cap I_{2}\right)$ is at most $2^{79 n / 200} 3^{n / 400} \cdot 2^{2 n / 5}=2^{159 n / 200} 3^{n / 400}$. In addition, since $\left|A_{1} \backslash I_{1}\right|+\left|A_{2} \backslash I_{2}\right| \leq \delta n$, there are at most $2^{2 H(\delta) n}$ choices for $\left(A_{1} \backslash I_{1}, A_{2} \backslash I_{2}\right)$. From 
these estimates it follows that there are at most $2^{159 n / 200} 3^{n / 400} \cdot 2^{2 H(\delta) n}=o\left(2^{4 n / 5}\right)$ possible assignments for $\left(A_{1}, A_{2}\right)$. The same conclusion can be drawn for the case that $A_{2}$ has at least one element in $\left[\left(\frac{2}{5}-\frac{1}{100}\right) n\right] \cup\left[\left(\frac{4}{5}+\frac{1}{100}\right) n, n\right]$.

From now on we may restrict our attention to those $A \in \mathrm{SF}_{2}(n)$ satisfying $(\alpha)$. Let

$$
S(A)=\{x \in A: x \leq n / 5\}
$$

denote the collection of elements of $A$ which are at most $n / 5$. We shall count the number of sets $A \in \mathrm{SF}_{2}(n)$ with $S(A)$ fixed. The following simple but crucial observation will be exploited several times to bound the number of ways to choose $A \cap\{n / 5+1, \ldots, n\}$.

Observation. Every set $A \in \mathrm{SF}_{2}(n)$ with property $(\alpha)$ satisfies the following:

(i) $S(A)=A_{1} \cap\left[\left(\frac{1}{5}-\frac{1}{100}\right) n, \frac{1}{5} n\right]$, and $A \cap(S(A)+S(A)) \subseteq A_{2} \cap\left[\left(\frac{2}{5}-\frac{1}{50}\right) n, \frac{2}{5} n\right]$;

(ii) If $X \subseteq A_{2} \cap\left[\left(\frac{2}{5}-\frac{1}{50}\right) n, \frac{2}{5} n\right]$, then $A \cap\{4 n / 5+1, \ldots, n\}$ and $S+(A \cap 2 X)$ are disjoint subsets of $\{4 n / 5+1, \ldots, n\}$.

Proof. To ease notation we shall write $S$ for $S(A)$.

(i) The first statement holds since $A_{2} \cap[n / 5]=\emptyset$ and $\min \left(A_{1}\right) \geq\left(\frac{1}{5}-\frac{1}{100}\right) n$ by property $(\alpha)$. Since $S=A_{1} \cap\left[\left(\frac{1}{5}-\frac{1}{100}\right) n, \frac{1}{5} n\right]$, we have $2 S \subseteq 2 A_{1} \cap\left[\left(\frac{2}{5}-\frac{1}{50}\right) n, \frac{2}{5} n\right]$. As $A_{1} \cap 2 A_{1}=\emptyset$ and $A=A_{1} \cup A_{2}$, this forces

$$
(A \cap 2 S) \subseteq\left(A \cap 2 A_{1}\right) \cap\left[\left(\frac{2}{5}-\frac{1}{50}\right) n, \frac{2}{5} n\right] \subseteq A_{2} \cap\left[\left(\frac{2}{5}-\frac{1}{50}\right) n, \frac{2}{5} n\right] .
$$

(ii) As $X \subseteq A_{2} \cap\left[\left(\frac{2}{5}-\frac{1}{50}\right) n, \frac{2}{5} n\right]$, we have $2 X \subseteq 2 A_{2} \cap\left[\left(\frac{4}{5}-\frac{1}{25}\right) n, \frac{4}{5} n\right]$. Since $A_{2} \cap 2 A_{2}=\emptyset$ and $A=A_{1} \cup A_{2}$, it follows that

$$
(A \cap 2 X) \subseteq\left(A \cap 2 A_{2}\right) \cap\left[\left(\frac{4}{5}-\frac{1}{25}\right) n, \frac{4}{5} n\right] \subseteq A_{1} \cap\left[\left(\frac{4}{5}-\frac{1}{25}\right) n, \frac{4}{5} n\right] .
$$

As $S=A_{1} \cap\left[\left(\frac{1}{5}-\frac{1}{100}\right) n, \frac{1}{5} n\right]$ due to (i), this implies $S+(A \cap 2 X) \subseteq 2 A_{1} \cap\left[\left(1-\frac{1}{20}\right) n, n\right]$. In particular, one has $S+(A \cap 2 X) \subseteq\{4 n / 5+1, \ldots, n\}$. Furthermore, the intersection of $A$ and $S+(A \cap 2 X)$ is contained in $\left(A \cap 2 A_{1}\right) \cap\left[\left(1-\frac{1}{20}\right) n, n\right] \subseteq A_{2} \cap\left[\left(1-\frac{1}{20}\right) n, n\right] \stackrel{(\alpha)}{=} \emptyset$. These properties imply the statement.

The remainder of the proof involves some careful counting using the observation as well as Lemmas 3.7, 3.8 and 3.9. We shall break up the calculation into three claims. In the first two, we count the sets $A$ for which $\sum_{a \in S(A)}(n / 5-a)$ is large (Claim 3.13), or $\sum_{a \in S(A)}(n / 5-a)$ is small and $|S(A)+S(A)|$ is large (Claim 3.14). Finally we count the remaining sets in Claim 3.15 .

Let $\mathcal{S}(k, \ell)$ denote the collection of sets $S \subseteq[n / 5]$ with $|S|=\ell$ and

$$
\sum_{a \in S}(n / 5-a)=k \text {. }
$$

Claim 3.13. For a given fixed $\ell \in \mathbb{N}$, there are at most $e^{-\ell} 2^{4 n / 5}$ sets $A \in \mathrm{SF}_{2}(n)$ of type $(b)$ which satisfy $(\alpha)$ and with $S(A) \in \mathcal{S}(k, \ell)$ for some $k \geq \ell^{2} / \delta^{2}$.

Proof. For $k \geq \ell^{2} / \delta^{2}$ and $S \in \mathcal{S}(k, \ell)$, let $\mathcal{I}(S)$ denote the family of all sets $A \in \mathrm{SF}_{2}(n)$ of type $(b)$ that satisfy $(\alpha)$ and with $S(A)=S$. We shall first bound $\mathcal{I}(S)$ and then sum over choices of $S$. Define the graph $G$ of 'forbidden monochromatic pairs' by setting

$$
V(G)=\{n / 5+1, \ldots, 2 n / 5\} \text {, and } E(G)=\{\{x, x+s\}: s \in S\} .
$$

We partition $\mathcal{I}(S)=\mathcal{I}_{1}(S) \cup \mathcal{I}_{2}(S)$, in which $\mathcal{I}_{1}(S)$ consists of all those sets $A \in \mathcal{I}(S)$ having the property that $A \cap V(G)$ contains at most $k / 8$ edges of $G$. 
We shall use Janson Inequality to estimate $\left|\mathcal{I}_{1}(S)\right|$. Observe that $G$ has $k$ edges and maximum degree at most $2 \ell$, since $S(A)=S \in \mathcal{S}(k, \ell)$. Let $\mu$ and $\Delta$ be the quantities defined in the statement of Lemma 3.9 and note that we are applying the lemma with $|\Gamma|=n / 5$. We have

$$
\mu=k \cdot\left(\frac{1}{2}\right)^{2}=k / 4 \quad \text { and } \quad \Delta \leq 4 k \ell \cdot\left(\frac{1}{2}\right)^{3}=k \ell / 2 .
$$

Accordingly $\mu^{2} /(8 \mu+8 \Delta) \geq k /(96 \ell)$, and so the number of choices for $A \cap V(G)$ is at most $e^{-k / 96 \ell} 2^{n / 5}$. On the other hand, we can pick $A \cap\{2 n / 5+1, \ldots, n\}$ in at most $2^{3 n / 5}$ ways. We thus have

$$
\left|\mathcal{I}_{1}(S)\right| \leq e^{-k / 96 \ell} 2^{n / 5} \cdot 2^{3 n / 5}=e^{-k / 96 \ell} 2^{4 n / 5} .
$$

We proceed to bound $\left|\mathcal{I}_{2}(S)\right|$. For each subset $T \subseteq V(G)$ so that $T$ contains at least $k / 8$ edges of $G$, we define $\mathcal{I}_{2}(S, T)$ to be the collection of sets $A \in \mathcal{I}_{2}(S)$ with $A \cap V(G)=T$. We see immediately that $\mathcal{I}_{2}(S)=\bigcup_{T} \mathcal{I}_{2}(S, T)$, and so the task is now to estimate $\left|\mathcal{I}_{2}(S, T)\right|$. Observe that a set $A \in \mathcal{I}_{2}(S, T)$ is uniquely determined by the intersection of $A$ and $\{2 n / 5+1, \ldots, n\}$. For this reason we fix $S$ and $T$, and bound the number of ways to choose $A \cap\{2 n / 5+1, \ldots, n\}$. Since $G$ has maximum degree at most $2 \ell$, we may select $k / 16 \ell$ disjoint edges in $T=A \cap V(G)$, say $\left\{x_{i}, x_{i}+s_{i}\right\}$ with $1 \leq i \leq k / 16 \ell$. Let $B=\left\{x_{i}+s_{i}: 1 \leq i \leq k / 16 \ell\right\}$. Then, $s_{i} \in A_{1} \cap\left[\left(\frac{1}{5}-\frac{1}{100}\right) n, \frac{1}{5} n\right]$ by Observation (i), and so $x_{i} \in A_{1} \cap\left[\frac{1}{5} n,\left(\frac{1}{5}+\frac{1}{100}\right) n\right]$ due to property $(\alpha)$ and the fact that $x_{i}+s_{i} \leq 2 n / 5$. Hence $B \subseteq 2 A_{1} \cap\left[\left(\frac{2}{5}-\frac{1}{100}\right) n, \frac{2}{5} n\right]$. Since $A_{1}$ is sum-free and $B \subseteq A$, this forces

We thus have

$$
B \subseteq A_{2} \cap\left[\left(\frac{2}{5}-\frac{1}{100}\right) n, \frac{2}{5} n\right] .
$$

$$
2 B \subseteq\left[\left(\frac{4}{5}-\frac{1}{50}\right) n, \frac{4}{5} n\right], \text { and }|2 B| \geq 2|B|-1 \geq k / 16 \ell .
$$

This suggests us splitting $\mathcal{I}_{2}(S, T)=\mathcal{I}_{2}^{\prime}(S, T) \cup \mathcal{I}_{2}^{\prime \prime}(S, T)$, where $\mathcal{I}_{2}^{\prime}(S, T)$ contains every set $A \in \mathcal{I}_{2}(S, T)$ with $|A \cap 2 B| \leq|2 B| / 4$.

We consider a set $A \in \mathcal{I}_{2}^{\prime}(S, T)$. Since $|A \cap 2 B| \leq|2 B| / 4$ by the definition of $\mathcal{I}_{2}^{\prime}(S, T)$, we may pick $A \cap 2 B$ from the family of all subsets of $2 B$ in at most $2^{H(1 / 4)|2 B|}$ ways. Thus, noting that $2 B \subseteq\{2 n / 5+1, \ldots, n\}$ by (3.4) and that $A$ is determined by $A \cap\{2 n / 5+1, \ldots, n\}$, we have

$$
\left|\mathcal{I}_{2}^{\prime}(S, T)\right| \leq 2^{H(1 / 4)|2 B|} \cdot 2^{3 n / 5-|2 B|} \stackrel{(3.4)}{\leq} 2^{3 n / 5-k / 90 \ell} .
$$

Suppose now that $A \in \mathcal{I}_{2}^{\prime \prime}(S, T)$. Evidently there are at most $2^{2 n / 5}$ ways to choose $A \cap\{2 n / 5+1, \ldots, 4 n / 5\}$. We shall fix this set and bound the number of possibilities for $A \cap\{4 n / 5+1, \ldots, n\}$. As $2 B \subseteq\{2 n / 5+1, \ldots, 4 n / 5\}$ by (3.4), $S+(A \cap 2 B)$ is already determined. Moreover, it follows from property (3.3) and Observation (ii) that $A \cap\{4 n / 5+1, \ldots, n\}$ and $S+(A \cap 2 B)$ are two disjoint subsets of $\{4 n / 5+1, \ldots, n\}$. Hence there are at most $2^{n / 5-|S+(A \cap 2 B)|} \leq 2^{n / 5-|A \cap 2 B|}$ possible outcomes for the set $A \cap\{4 n / 5+1, \ldots, n\}$. Therefore, we get the estimate

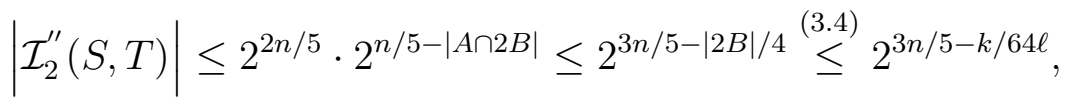

in which the second inequality follows from the definition of $\mathcal{I}_{2}^{\prime \prime}(S, T)$. 
Combining inequalities (3.5) and (3.6) gives

$$
\left|\mathcal{I}_{2}(S)\right|=\sum_{T \subseteq\{n / 5+1, \ldots 2 n / 5\}}\left(\left|\mathcal{I}_{2}^{\prime}(S, T)\right|+\left|\mathcal{I}_{2}^{\prime \prime}(S, T)\right|\right) \leq 2^{1-k / 90 \ell} 2^{4 n / 5}
$$

Finally there are at most $\left(\frac{e^{2} k}{\ell^{2}}\right)^{\ell}$ choices for $S \in \mathcal{S}(k, \ell)$ by Lemma 3.8, and hence, using (3.2) and (3.7), we can bound the number of sets $A$ from above by

$$
\begin{aligned}
\sum_{k \geq \ell^{2} / \delta^{2}} \sum_{S \in \mathcal{S}(k, \ell)}\left(e^{-k / 96 \ell}+2^{1-k / 90 \ell}\right) 2^{4 n / 5} & \leq \sum_{k \geq \ell^{2} / \delta^{2}} 4 \cdot\left(\frac{e^{2} k}{\ell^{2}}\right)^{\ell} e^{-k / 130 \ell} 2^{4 n / 5} \\
& \leq 1040 \ell\left(\frac{e^{2}}{\delta^{2}}\right)^{\ell} e^{-\ell / 130 \delta^{2}} 2^{4 n / 5} \leq e^{-\ell} 2^{4 n / 5}
\end{aligned}
$$

where the second inequality holds since $g(x)=x^{a} e^{-b x}$ is decreasing on $[a / b, \infty)$ and $g(x+1 / b)<g(x) / 2$ for $x \geq 4 a / b$. (Note that we have $\ell^{2} / \delta^{2} \geq 4 \ell \cdot 130 \ell$ since $\delta>0$ is sufficiently small.)

Claim 3.14. For a given fixed $\ell \in \mathbb{N}$, there are at most $e^{-\ell} 2^{4 n / 5}$ sets $A \in \mathrm{SF}_{2}(n)$ of type $(b)$ that satisfy $(\alpha)$ and with

$\left(\beta_{1}\right) S(A) \in \mathcal{S}(k, \ell)$ for some $k \leq \ell^{2} / \delta^{2}$;

$\left(\beta_{2}\right)|S(A)+S(A)| \geq|S(A)| / \delta^{2}$.

Proof. The proof is similar in spirit to that of Claim 3.13. Fixing an integer $k$ with $k \leq \ell^{2} / \delta^{2}$ and a set $S \in \mathcal{S}(k, \ell)$ with $|2 S| \geq \ell / \delta^{2}$, we denote by $\mathcal{I}(S)$ the collection of all sets $A \in \mathrm{SF}_{2}(n)$ of type $(b)$ that satisfy $(\alpha)$ and with $S(A)=S$. Further partition $\mathcal{I}(S)=\mathcal{I}_{1}(S) \cup \mathcal{I}_{2}(S)$, where $\mathcal{I}_{1}(S)$ consists of all sets $A \in \mathcal{I}(S)$ with $|A \cap 2 S| \leq|2 S| / 4$.

We first count $\mathcal{I}_{1}(S)$. Notice that $2 S \subseteq\{n / 5+1, \ldots, 2 n / 5\}$ due to Observation (i), and $|A \cap 2 S| \leq|2 S| / 4$ by the definition of $\mathcal{I}_{1}(S)$. From this we deduce that there are no more than $2^{n / 5-|2 S|} 2^{H(1 / 4)|2 S|}$ choices for $A \cap\{n / 5+1, \ldots, 2 n / 5\}$. Since we can take $A \cap\{2 n / 5+1, \ldots, n\}$ in at most $2^{3 n / 5}$ possible ways, it follows that

$$
\left|\mathcal{I}_{1}(S)\right| \leq 2^{n / 5-|2 S|} 2^{H(1 / 4)|2 S|} \cdot 2^{3 n / 5} \leq 2^{4 n / 5-\ell / 6 \delta^{2}}
$$

for $|2 S| \geq \ell / \delta^{2}$.

We next deal with $\mathcal{I}_{2}(S)$. For each subset $T \subseteq 2 S$ with $|T| \geq|2 S| / 4$, we define $\mathcal{I}_{2}(S, T)$ to be the collection of sets $A \in \mathcal{I}_{2}(S)$ with $A \cap 2 S=T$. We shall fix such a set $T$ and further partition $\mathcal{I}_{2}(S, T)=\mathcal{I}_{2}^{\prime}(S, T) \cup \mathcal{I}_{2}^{\prime \prime}(S, T)$, in which $\mathcal{I}_{2}^{\prime}(S, T)$ consists of sets $A \in \mathcal{I}_{2}(S, T)$ with $|A \cap 2 T| \leq|2 T| / 4$. Note that

$$
|2 T| \geq|T| \geq|2 S| / 4 \geq \ell / 4 \delta^{2} .
$$

Suppose first that $A \in \mathcal{I}_{2}^{\prime}(S, T)$. Then $|A \cap 2 T| \leq|2 T| / 4$ by the definition of $\mathcal{I}_{2}^{\prime}(S, T)$, and so we can choose $A \cap 2 T$ in at most $2^{H(1 / 4)|2 T|}$ ways. Moreover, by Observation (i) we have $2 S \subseteq\{n / 5+1, \ldots, 2 n / 5\}$ and $2 T \subseteq 4 S \subseteq\{2 n / 5+1, \ldots, 4 n / 5\}$. So there are at most $2^{4 n / 5-|2 S|-|2 T|}$ possibilities for $A \backslash(S \cup 2 S \cup 2 T)$. (Recall that $S=A \cap[n / 5]$.) We therefore obtain

$$
\left|\mathcal{I}_{2}^{\prime}(S, T)\right| \leq 2^{H(1 / 4)|2 T|} \cdot 2^{4 n / 5-|2 S|-|2 T|} \stackrel{(3.9)}{\leq} 2^{4 n / 5-|2 S|-\ell / 22 \delta^{2}} .
$$

Suppose now that $A \in \mathcal{I}_{2}^{\prime \prime}(S, T)$. Since $2 S \subseteq\{n / 5+1, \ldots, 2 n / 5\}$ by Observation (i), and the sets $S=A \cap[n / 5]$ and $T=A \cap 2 S$ have been chosen, we see that $A$ is uniquely 
determined by $A \cap(\{n / 5+1, \ldots, 4 n / 5\} \backslash 2 S)$ and $A \cap\{4 n / 5+1, \ldots, n\}$. We can trivially bound the number of choices for $A \cap(\{n / 5+1, \ldots, 4 n / 5\} \backslash 2 S)$ by $2^{3 n / 5-|2 S|}$. We shall fix this set and bound the number of ways to choose $A \cap\{4 n / 5+1, \ldots, n\}$. Note that fixing $A \cap[4 n / 5]$ determines $S+(A \cap 2 T)$. Furthermore, we know from Observation (i) that $T=A \cap 2 S$ is contained in $A_{2} \cap\left[\left(\frac{2}{5}-\frac{1}{50}\right) n, \frac{2}{5} n\right]$, and consequently $A \cap\{4 n / 5+1, \ldots, n\}$ and $S+(A \cap 2 T)$ are disjoint subsets of $\{4 n / 5+1, \ldots, n\}$ due to Observation (ii). Hence we can assign $A \cap\{4 n / 5+1, \ldots, n\}$ in at most $2^{n / 5-|S+(A \cap 2 T)|} \leq 2^{n / 5-|A \cap 2 T|}$ possible ways, as $S \neq \emptyset$. Putting everything together we get

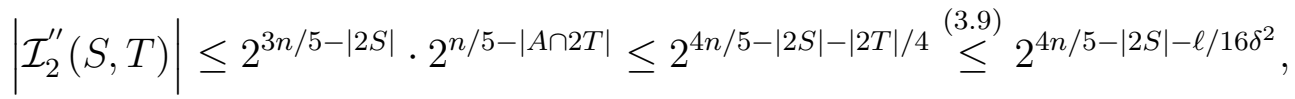

where the second inequality holds since $|A \cap 2 T| \geq|2 T| / 4$ by the definition of $\mathcal{I}_{2}^{\prime \prime}(S, T)$.

Using inequalities (3.10) and (3.11) yields

$$
\left|\mathcal{I}_{2}(S)\right|=\sum_{T \subseteq 2 S}\left(\left|\mathcal{I}_{2}^{\prime}(S, T)\right|+\left|\mathcal{I}_{2}^{\prime \prime}(S, T)\right|\right) \leq 2^{1-\ell / 22 \delta^{2}} 2^{4 n / 5} .
$$

Finally adding inequalities (3.8) and (3.12), and summing over all $S$, we get the following bound on the number of sets $A$ :

$$
\begin{aligned}
\sum_{k \leq \ell^{2} / \delta^{2}} \sum_{S \in \mathcal{S}(k, \ell)}\left(2^{-\ell / 6 \delta^{2}}+2^{1-\ell / 22 \delta^{2}}\right) 2^{4 n / 5} & \leq \sum_{k \leq \ell^{2} / \delta^{2}}\left(\frac{e^{2} k}{\ell^{2}}\right)^{\ell} 2^{2-\ell / 22 \delta^{2}} 2^{4 n / 5} \\
& \leq \frac{\ell^{2}}{\delta^{2}} \cdot\left(\frac{e^{2}}{\delta^{2}}\right)^{\ell} 2^{2-\ell / 22 \delta^{2}} 2^{4 n / 5} \leq e^{-\ell} 2^{4 n / 5}
\end{aligned}
$$

where the first inequality holds since $|\mathcal{S}(k, \ell)| \leq\left(\frac{e^{2} k}{\ell^{2}}\right)^{\ell}$ due to Lemma 3.8

The following claim now completes the proof of Lemma 3.11.

Claim 3.15. There exists an absolute constant $\ell_{0}$ so that for every integer $\ell \geq \ell_{0}$ there are at most $e^{-\ell / 5} 2^{4 n / 5}$ sets $A \in \mathrm{SF}_{2}(n)$ of type (b) which satisfy $(\alpha)$ and with

$\left(\gamma_{1}\right) S(A) \in \mathcal{S}(k, \ell)$ for some $k \leq \ell^{2} / \delta^{2}$;

$\left(\gamma_{2}\right)|S(A)+S(A)| \leq|S(A)| / \delta^{2}$.

Proof. This is the most difficult case, and we shall have to count more carefully, using Lemma 3.7. For each $k \in \mathbb{N}$ and $\lambda>0$, let $\mathcal{S}_{(\lambda)}(k, \ell)$ denote the collection of sets $S \in \mathcal{S}(k, \ell)$ such that

$$
\lambda|S| \leq|2 S| \leq(1+\delta) \lambda|S|
$$

Given $S \in \mathcal{S}_{(\lambda)}(k, \ell)$, we denote by $\mathcal{I}(S)$ the collection of all sets $A \in \mathrm{SF}_{2}(n)$ of type $(b)$ that satisfy $(\alpha)$ and with $S(A)=S$. It is not hard to see that the number of sets $A$ that satisfies the hypothesis of Claim 3.15 is bounded from above by $\sum|\mathcal{I}(S)|$, where the sum is taken over all triples $(k, \lambda, S)$ with $k \leq \ell^{2} / \delta^{2}, \lambda=(2-\delta)(1+\delta)^{i}$ for some integer $i$ with $0 \leq i \leq \frac{3}{\delta} \ln \frac{1}{\delta}$, and $S \in \mathcal{S}_{(\lambda)}(k, \ell)$. To count $\mathcal{I}(S)$, we partition $\mathcal{I}(S)=\mathcal{J}(S) \cup \mathcal{K}(S)$, in which $\mathcal{J}(S)$ consists of all sets $A \in \mathcal{I}(S)$ with $|A \cap 2 S| \leq|2 S| / 20$.

We shall use Lemma 3.7 to count the number of triples $(k, \lambda, S)$. As noted above, there are only $O_{\delta}\left(\ell^{2}\right)$ choices for $k$ and $\lambda$; this will be absorbed by the error term $2^{O(\delta \ell)}$. We may apply Lemma 3.7 to $R=(1+\delta) \lambda, s=\ell$ and $D=k$, and conclude that there are at most $2^{O(\delta \ell)}\left(\begin{array}{c}(1+\delta) \lambda \ell / 2 \\ \ell\end{array}\right)$ choices for $S \in \mathcal{S}_{(\lambda)}(k, \ell)$. (Note that $(1+\delta) \lambda=O_{\delta}(1)$, $k=O_{\delta}\left(\ell^{2}\right)$ and $\ell$ is sufficiently large.) 
We are now ready to estimate the sum $\sum_{(k, \lambda, S)}|\mathcal{J}(S)|$. Analysis similar to that in the proof of Claim 3.14 shows

$$
|\mathcal{J}(S)| \leq 2^{4 n / 5-|2 S|} 2^{H(1 / 20)|2 S|} \leq 2^{4 n / 5-2 \lambda \ell / 3}
$$

since $|2 S| \geq \lambda \ell$ for all $S \in \mathcal{S}_{(\lambda)}(k, \ell)$. Summing over all choices of $(k, \lambda, S)$, and recalling that $\lambda=(2-\delta)(1+\delta)^{i} \geq 2-\delta$, we thus get

$$
\begin{aligned}
\sum_{(k, \lambda, S)}|\mathcal{J}(S)| & \leq \sum_{\lambda} 2^{O(\delta \ell)}\left(\begin{array}{c}
(1+\delta) \lambda \ell / 2 \\
\ell
\end{array}\right) 2^{4 n / 5-2 \lambda \ell / 3} \\
& \leq \sum_{\lambda} 2^{O(\delta \ell)} 2^{(1+\delta) \lambda \ell / 2} 2^{4 n / 5-2 \lambda \ell / 3} \leq 2^{4 n / 5-0.33 \ell}
\end{aligned}
$$

We proceed to bound the sum $\sum_{(k, \lambda, S)}|\mathcal{K}(S)|$. For each $p \in \mathbb{N}$ and $\mu>0$, let $\mathcal{T}^{(\mu)}(S, p)$ be the collection of sets $T \subseteq 2 S$ with

$$
|T|=p \text {, and } \mu|T| \leq|2 T| \leq(1+\delta) \mu|T| .
$$

For any set $T \in \mathcal{T}^{(\mu)}(S, p)$ and any integer $q$ with $0 \leq q \leq|2 T|$, let $\mathcal{K}(T, q)$ stand for the collection of those sets $A \in \mathcal{K}(S)$ with $A \cap 2 S=T$ and $|A \cap 2 T|=q$. From the definition of $\mathcal{K}(S)$, we know that $|2 S| / 20 \leq|T| \leq|2 S|$ (otherwise $\mathcal{K}(T, q)=\emptyset$ ), and so $\lambda \ell / 20 \leq p \leq(1+\delta) \lambda \ell$. Moreover, as $|2 S| \leq|S| / \delta^{4}$ by our choice of $\lambda$, Lemma 3.6 implies $|4 S| \leq|S| / \delta^{16}$, giving $|2 T| \leq|4 S| \leq|2 S| / \delta^{16} \leq 20|T| / \delta^{16}$. Accordingly we only need to care about those $\mu$ so that $\mu=(2-\delta)(1+\delta)^{j}$ for some integer $j$ with $0 \leq j \leq \frac{17}{\delta} \ln \frac{1}{\delta}$. Summarizing, we have

$$
|\mathcal{K}(S)| \leq \sum_{(p, \mu, T, q)}|\mathcal{K}(T, q)|
$$

where the sum is over all quadruples $(p, \mu, T, q)$ such that $\lambda \ell / 20 \leq p \leq(1+\delta) \lambda \ell$, $\mu=(2-\delta)(1+\delta)^{j}$ for some integer $j$ with $0 \leq j \leq \frac{17}{\delta} \ln \frac{1}{\delta}, T \in \mathcal{T}^{(\mu)}(S, p)$, and $q \leq|2 T|$.

From the previous discussion, we deduce that there are only $O_{\delta}(p)$ choices for $p$ and $\mu$; this will be absorbed by the error term $2^{O(\delta p)}$. Using Lemma 3.7 with $R=(1+\delta) \mu, s=p$ and $D=2 k$, we find that there are at most $2^{O(\delta p)}\left(\begin{array}{c}(1+\delta) \mu p / 2 \\ p\end{array}\right)$ choices for $T \in \mathcal{T}^{(\mu)}(S, p)$. Since $q \leq|2 T| \leq(1+\delta) \mu p$, we have only $O_{\delta}(p)$ possibilities for $q$, and this will also be absorbed by the error term $2^{O(\delta p)}$.

We are reduced to enumerating $\mathcal{K}(T, q)$ for fixed $T \in \mathcal{T}^{(\mu)}(S, p)$ and $q \leq(1+\delta) \mu p$. Since $|A \cap 2 T|=q$, there are at most $\left(\begin{array}{c}|2 T| \\ q\end{array}\right)$ choices for $A \cap 2 T$. In addition, since $2 S$ and $2 T$ are disjoint subsets of $\{2 n / 5+1, \ldots, 4 n / 5\}$ due to Observation (i), we can allocate $A \cap(\{2 n / 5+1, \ldots, n\} \backslash(2 S \cup 2 T))$ in at most $2^{3 n / 5-|2 S|-|2 T|}$ possible ways. Furthermore, specifying $A \cap[4 n / 5]$ determines $S+(A \cap 2 T)$. As $A \cap\{4 n / 5+1, \ldots, n\}$ and $S+(A \cap 2 T)$ are disjoint subsets of $\{4 n / 5+1, \ldots, n\}$ by Observation (ii), this implies that there are at most $2^{n / 5-|S+(A \cap 2 T)|} \leq 2^{n / 5-q}$ possibilities for $A \cap\{4 n / 5+1, \ldots, n\}$. Here we evaluate $|S+(A \cap 2 T)| \geq|A \cap 2 T|=q$ for $S \neq \emptyset$. Therefore, recalling that $|2 S| \geq \lambda \ell$ and $\mu p \leq|2 T| \leq(1+\delta) \mu p$, we get

$$
|\mathcal{K}(T, q)| \leq\left(\begin{array}{c}
|2 T| \\
q
\end{array}\right) \cdot 2^{3 n / 5-|2 S|-|2 T|} \cdot 2^{n / 5-q} \leq\left(\begin{array}{c}
(1+\delta) \mu p \\
q
\end{array}\right) 2^{4 n / 5-\lambda \ell-\mu p-q} .
$$


From what has already been proved we may bound $\sum_{(k, \lambda, S)}|\mathcal{K}(S)|$ from above by

$$
\begin{aligned}
& \sum_{(k, \lambda, S)} \sum_{(p, \mu, T, q)}|\mathcal{K}(T, q)| \\
& \leq \sum_{(\lambda, p, \mu, q)} 2^{O(\delta \ell)}\left(\begin{array}{c}
(1+\delta) \lambda \ell / 2 \\
\ell
\end{array}\right) \cdot 2^{O(\delta p)}\left(\begin{array}{c}
(1+\delta) \mu p / 2 \\
p
\end{array}\right) \cdot\left(\begin{array}{c}
(1+\delta) \mu p \\
q
\end{array}\right) 2^{4 n / 5-\lambda \ell-\mu p-q} \\
& \leq 2^{4 n / 5} \cdot \max _{(\lambda, p, \mu, q)}\left\{\left(\begin{array}{c}
(1+\delta) \lambda \ell / 2 \\
\ell
\end{array}\right) 2^{-\lambda \ell} \cdot 2^{O(\delta p)}\left(\begin{array}{c}
(1+\delta) \mu p / 2 \\
p
\end{array}\right) 2^{-\mu p} \cdot\left(\begin{array}{c}
(1+\delta) \mu p \\
q
\end{array}\right) 2^{-q}\right\},
\end{aligned}
$$

where in the last inequality we used the fact that the term $2^{O(\delta \ell)}$ is absorbed by the error term $2^{O(\delta p)}$. We shall deploy the entropy estimate (3.1) to control the last expression. For abbreviation, set $x=(1+\delta) \lambda / 2, y=(1+\delta) \mu / 2, z=q /(1+\delta) \mu p$, and $\mathbb{D}=$ $\left\{(x, p, y, z) \in \mathbb{R}^{4}: x \geq 1,0 \leq p \leq 2 x \ell, y \geq 1,0 \leq z \leq 1\right\}$. Recalling that $\lambda, \mu \geq 2-\delta$, $0 \leq p \leq(1+\delta) \lambda \ell$ and $0 \leq q \leq(1+\delta) \mu p$, we then have $(x, p, y, z) \in \mathbb{D}$. Now using inequality (3.1) and simplifying yields

$$
\sum_{(k, \lambda, S)}|\mathcal{K}(S)| \leq 2^{4 n / 5} \cdot \max _{(x, p, y, z) \in \mathbb{D}} 2^{h(x, p, y, z)}
$$

where $h(x, p, y, z):=\left(x H\left(\frac{1}{x}\right)-\frac{2 x}{1+\delta}\right) \ell+\left(y H\left(\frac{1}{y}\right)-\frac{2 y}{1+\delta}+O(\delta)\right) p+2 y p \cdot(H(z)-z)$. A straightforward but slightly tedious calculation shows that the maximum value of $h(x, p, y, z)$ on $\mathbb{D}$ is $\left(\log _{2}\left(\frac{81}{115}\right)+O(\delta)\right) \ell \approx-0.505 \ell$, attained at $z=\frac{1}{3}, y=\frac{16}{7}+O(\delta)$, $p=2 x \ell$ and $x=\frac{196}{115}+O(\delta)$ Hence

$$
\sum_{(k, \lambda, S)}|\mathcal{K}(S)| \leq 2^{4 n / 5} \cdot 2^{-0.5 \ell}=2^{4 n / 5-0.5 \ell}
$$

Finally adding inequalities (3.13) and (3.14), and summing over all triples $(k, \lambda, S)$, we conclude that the number of sets $A$ is at most

$$
\sum_{(k, \lambda, S)}|\mathcal{I}(S)| \leq \sum_{(k, \lambda, S)}(|\mathcal{J}(S)|+|\mathcal{K}(S)|) \leq\left(2^{-0.33 \ell}+2^{-0.5 \ell}\right) 2^{4 n / 5} \leq e^{-\ell / 5} 2^{4 n / 5}
$$

The proof of Lemma 3.11 is at long last complete.

\section{Concluding Remarks}

In this paper we studied the general structure of large sum-free sets of integers. From this we obtained a good bound on the total number of 2-wise sum-free subsets of $[n]$. It is likely that our methods extend to give an asymptotic formula for this number, but we do not pursue this here. We close with some remarks and possible directions for further research.

\footnotetext{
${ }^{4}$ We can solve this optimisation problem backwardly using the following simple facts. Firstly, the function $f(z)=H(z)-z$ achieves its maximum at $z=1 / 3$. Secondly, given $\rho>0$, the function $g(t)=t H\left(\frac{1}{t}\right)-\rho t$ is maximised at $t=2^{\rho} /\left(2^{\rho}-1\right)$.
} 
Sets with small difference constant. The main open problem is to determine the critical density threshold at which Theorem 1.4 ceases to hold. Note that in the theorem, the value for $c$ given by our argument is something like $10^{-6} q_{2.6}^{2}$, where $q_{2.6}$ is the absolute positive constant from Jin's inverse theorem (Lemma [2.6). Note that Jin obtained his result via non-standard analysis, and thus no explicit value of 22.6 can be extracted from his proof. Using the following conjecture instead of Lemma [2.6, we would certainly get a reasonable value for $c$.

Conjecture 4.1. There exists a natural number $K$ such that for any finite set of integers $A$ so that $|A| \geq K$ and $|A-A|=3|A|-3+r$ for some integer $r$ with $0<r<\frac{1}{3}|A|-2$, one of the following properties holds:

(i) $A$ is a subset of an arithmetic progression of length $2|A|-1+2 r$;

(ii) $A \subseteq P_{1} \cup P_{2}$ for some arithmetic progressions $P_{1}, P_{2}$ with common step and $\left|P_{1}\right|+\left|P_{2}\right| \leq|A|+r$.

We remark that the sumset version of Conjecture 4.1 was proposed by Freiman [24]. The following example shows that the condition $r<\frac{1}{3}|A|-2$ is necessary.

Example 4.2. Let $y \geq 4 x$, and consider the set $A=\{0, y, 2 y\}+[0, x-1]$. We have $A-A=\{0, \pm y, \pm 2 y\}+[-x+1, x-1]$, and so $|A-A|=10 x-5=(3|A|-3)+\left(\frac{1}{3}|A|-2\right)$. But $A$ is neither a subset of an arithmetic progression of length $(2|A|-1)+2 \cdot\left(\frac{1}{3}|A|-2\right)$ nor a subset of an union of two arithmetic progressions of total length $|A|+\left(\frac{1}{3}|A|-2\right)$.

It is worth mentioning that Eberhard, Green and Manners 17 provided a rough structure theorem for sets of integers of difference constant less than 4. Specifically, they proved that if $A$ is a subset of $\mathbb{Z}$ with $|A-A| \leq(4-\epsilon)|A|$ then $A$ has density at least $\frac{1}{2}+2^{-1000} \epsilon$ on some arithmetic progression of length $\gg_{\epsilon}|A|$. They then used this result to show the existence of a set of $n$ positive integers with no sum-free subset of size greater than $\frac{1}{3} n+o(n)$, answering a famous question of Erdős [19] from 1965 .

Union of intersecting families. One can pursue the following general questions for any monotone property $\mathcal{P}$ :

(i) What is the maximum size of a union of $r$ objects with property $\mathcal{P}$ ?

(ii) How many objects which can be partitioned into $r$ subobjects having property $\mathcal{P}$ are there?

In this paper, we addressed the second question for the sum-free property. In what follows, we shall single out another monotone property for further research.

A family of sets is called intersecting if it does not contain two disjoint sets. Given a positive integer $r$, a family $\mathcal{F}$ is said to be $r$-wise intersecting if there exists a partition of $\mathcal{F}$ into $r$ intersecting families. Let $\mathcal{I}_{r}(n, k)$ denote the collection of all $r$ wise intersecting families $\mathcal{F} \subseteq\left(\begin{array}{c}{[n]} \\ k\end{array}\right)$. The celebrated Erdős-Ko-Rado theorem from 1961 states that for $n \geq 2 k$ the largest member of $\mathcal{I}_{1}(n, k)$ has size $\left(\begin{array}{l}n-1 \\ k-1\end{array}\right)$. Recently Ellis and Lifshitz [18] considered the problem, first raised by Erdős [20], of determining the maximum possible size of a family in $\mathcal{I}_{r}(n, k)$ when $r \geq 2$. Specifically, they showed $|\mathcal{F}| \leq\left(\begin{array}{l}n \\ k\end{array}\right)-\left(\begin{array}{c}n-r \\ k\end{array}\right)$ for any $\mathcal{F} \in \mathcal{I}_{r}(n, k)$ provided that $r \geq 2$ and $n \geq 2 k+C(r) k^{2 / 3}$, with equality holds if and only if $\mathcal{F}=\left\{F \in\left(\begin{array}{c}{[n]} \\ k\end{array}\right): F \cap R \neq \emptyset\right\}$ for some $R \in\left(\begin{array}{c}{[n]} \\ r\end{array}\right)$. In the case $r=2$, this significantly improves a previous result due to Frankl and Füredi [21]. It would be interesting to determine whether $C(r) k^{2 / 3}$ is the best possible error term. 
Note that an example given by Frankl and Füredi [21] shows that this term cannot be reduced to below $\sqrt{k}$.

The problem of enumerating $\mathcal{I}_{1}(n, k)$ was first investigated by Balogh, Das, Delcourt, Liu and Sharifzadeh [6]. Building on the of Balogh et al., Frankl and Kupavskii [22] and, independently, Balogh, Das, Liu, Sharifzadeh and Tran [7] established the asymptotic formula $\left|\mathcal{I}_{1}(n, k)\right|=(n+o(1)) 2^{\left(\begin{array}{c}n-1 \\ k-1\end{array}\right)}$ for $n \geq 2 k+3 \sqrt{k \ln k}$. Motivated by this result and the theorem of Ellis and Lifshitz, we make the following conjecture.

Conjecture 4.3. $\left|\mathcal{I}_{r}(n, k)\right|=\left(\left(\begin{array}{l}n \\ r\end{array}\right)+o(1)\right) 2^{\left(\begin{array}{c}n \\ k\end{array}\right)-\left(\begin{array}{c}n-r \\ k\end{array}\right)}$ for $r \geq 2$ and $n \geq 2 k+C(r) k^{0.9}$, where the term o(1) tends to 0 as $n \rightarrow \infty$.

\section{ACKNOWLEDGEMENT}

The author was supported by the Alexander Humboldt Foundation, and by the GACR grant GJ16-07822Y, with institutional support RVO:67985807. He would like to thank Jan Hladky and Phuong Dao for fruitful discussions. He is also grateful to an anonymous referee whose suggestions helped improve and clarify the manuscript.

\section{Appendix A. Missing proofs from Section 2}

In this appendix, we give the proofs of Lemmas 2.7, 2.10 and 2.13.

Proof of Lemma 2.7. (i) As $m \in A$, we must have $|A \cap\{i, m+i\}| \leq 1$ for all $i \in[u, v]$. Hence, by the union bound, we obtain

$$
|A \cap([u, v] \cup[u+m, v+m])| \leq \sum_{u \leq i \leq v}|A \cap\{i, m+i\}| \leq v-u+1 .
$$

(ii) Using part (i) with $v=u+m-1$, we find

$$
|A \cap[u, u+2 m-1]| \leq(u+m-1)-u+1=m .
$$

(iii) Write $v-u=2 k m+r$, where $k, r \in \mathbb{N}$ and $0 \leq r<2 m$. It follows easily from part (ii) that $|A \cap[u, u+2 k m-1]| \leq k m$. If $r \leq m-1$, then we can trivially evaluate

$$
|A \cap[u+2 k m, u+2 k m+r]| \leq r+1 \leq \frac{1}{2}(r+m+1) .
$$

If $m \leq r<2 m$, part (ii) gives $|A \cap[u+2 k m, u+2 k m+r]| \leq m<(r+m+1) / 2$. Hence in either case, we always have $|A \cap[u, v]| \leq k m+(r+m+1) / 2=(v-u+m+1) / 2$.

Proof of Lemma 2.10. We begin by showing that the set $[x, x+k-1] \backslash(A+B)$ has at most $2 \epsilon k$ elements for each integer $x \in\left[b_{1}, b_{\ell}+1\right]$. Indeed let $i \in[\ell]$ be the largest integer such that $b_{i} \leq x$. For convenience, set $b_{\ell+1}=b_{\ell}+1$. From the definition of $i$ and the fact that $b_{i+1}-b_{i} \leq k$, we find $[x, x+k-1] \subseteq\left[b_{i}, b_{i+1}+k-1\right]=\left\{b_{i}, b_{i+1}\right\}+[0, k-1]$. Moreover, since $|A| \geq(1-\epsilon) k$, there are at most $2 k \epsilon$ elements in $\left\{b_{i}, b_{i+1}\right\}+[0, k-1]$ which do not belong to $\left\{b_{i}, b_{i+1}\right\}+A$. Hence $[x, x+k-1]$ contains only elements of $A+B$, with at most $2 k \epsilon$ exceptions, as claimed.

Finally, because $\left[b_{1}, b_{\ell}+k\right]$ can be covered by at most $\left(k+b_{\ell}-b_{1}+1\right) / k+1$ intervals of the form $[x, x+k-1]$ with $x \in\left[b_{1}, b_{\ell}+1\right]$, we find

$$
|A+B| \geq\left(k+b_{\ell}-b_{1}+1\right)-2 k \epsilon \cdot\left(\frac{k+b_{\ell}-b_{1}+1}{k}+1\right) \geq(1-4 \epsilon)\left(k+b_{\ell}-b_{1}+1\right) .
$$


Proof of Lemma 2.13. (i) A proof of this result can be found in [15, Lemma 2.2].

(ii) Denote $s=|A|$. We wish to show that $x \in 2 A$ for each $x \in[2 k-2 s+2,2 s-2]$. Since $[2 k-2 s+2,2 s-2]=[k-s+1, s-1]+[k-s+1, s-1]$, one has $x=y+z$ for some integers $y, z \in[k-s+1, s-1]$. Note that $y+i, z-i \in[0, k]$ for every integer $i \in[-k+s-1, k-s+1]$, and $|[0, k] \backslash A| \leq k-s+1$. Thus by the pigeonhole principle, there exists $j \in[-k+s-1, k-s+1]$ so that $y+j, z-j \in A$. We then have $x=(y+j)+(z-j) \in 2 A$.

\section{REFERENCES}

[1] H. L. Abbott and E. T. H. Wang, Sum-free sets of integers, Proceedings of the American Mathematical Society 67 (1977), 11-16.

[2] N. Alon, Independent sets in regular graphs and sum-free subsets of abelian groups, Israel Journal of Mathematics 78 (1991), 247-256.

[3] N. Alon, J. Balogh, R. Morris and W. Samotij, Counting sum-free sets in abelian groups, Israel Journal of Mathematics 199 (2014), 309-344.

[4] N. Alon, J. Balogh, R. Morris and W. Samotij, A refinement of the Cameron-Erdös conjecture, Proceedings of the London Mathematical Society 108 (2014), 44-72.

[5] R. Balasubramanian, G. Prakash and D. S. Ramana, Sum-free subsets of finite abelian groups of type III, European Journal of Combinatorics 58 (2016), 181-202.

[6] J. Balogh, S. Das, M. Delcourt, H. Liu and M. Sharifzadeh, Intersecting families of dicrete structures are typically trivial, Journal of Combinatoral Theory. Series A 32 (2015), 224-245.

[7] J. Balogh, S. Das, H. Liu, M. Sharifzadeh and T. Tran, Structure and supersaturation for intersecting families, arXiv:1802.08018.

[8] J. Balogh, H. Liu, M. Sharifzadeh and A. Treglown, The number of maximal sum-free subsets of integers, Proceedings of the American Mathematical Society 143 (2015), 4713-4721.

[9] J. Balogh, H. Liu, M. Sharifzadeh and A. Treglown, Sharp bound on the number of maximal sum-free subsets of integers, Journal of the European Mathematical Society, to appear, arXiv:1502.07605

[10] J. Balogh, R. Morris and W. Samotij, Independent sets in hypergraphs, Journal of the American Mathematical Society 28 (2015), 669-709.

[11] N. J. Calkin, On the number of sum-free sets, Bulletin of the London Mathematical Society 22 (1990), 141-144.

[12] P. Cameron and P. Erdös, On the number of sets of integers with various properties, in Number Theory, Walter de Gruyter, Berlin, 1990, pp. 61-79.

[13] A. Davydov and L. Tombak, Quasi-perfect linear binary codes with distance 4 and complete caps in projective geometry, Problemy Peredachi Informatzii 25 (1989), 11-23.

[14] J. Deshouillers and G. Freiman, On sum-free sets modulo p, Functiones et Approximatio Commentarii Mathematici 35 (2006), 51-59.

[15] J. Deshouillers, G. Freiman, V. Sós and M. Temkin, On the structure of sum-free sets II, Astérisque 258 (1999), 149-161.

[16] J. Deshouillers and V. Lev, A refined bound for sumfree sets in groups of prime order, Bulletin of the London Mathematical Society 40 (2008), 863-875.

[17] S. Eberhard, B. Green and F. Manners, Sets of integers with no large sum-free subset, Annals of Mathematics 180 (2014), 621-652.

[18] D. Ellis and N. Lifshitz, On the union of intersecting families, arXiv:1610.03027.

[19] P. Erdös, Extremal problems in number theory, in Proceedings of Symposia in Pure Mathematics, Vol. 8, American Mathematical Society, Providence, RI, 1965, pp. 181-189.

[20] P. Erdős, Problems and results on finite and infinite combinatorial analysis, in Infinite and finite sets (Keszthely, 1973). Vol. I, Colloquia Mathematica Societatis János Bolyai, Vol. 10, NorthHolland, Amersterdam, 1975, pp. 403-424.

[21] P. Frankl and Z. Füredi, Extremal Problems concerning Kneser Graphs, Journal of Combinatorial Theory. Series B 40 (1986), 270-285. 
[22] P. Frankl and A. Kupavskii, Counting intersecting and pairs of cross-intersecting families, Combinatorics, Probability and Computing 27 (2018), 60-68.

[23] G. Freiman, The addition of finite sets. I, Izvestija Vysših Učebnyh Zavedeniu Matematika 6 (1959), 202-213.

[24] G. Freiman, Inverse problem of additive number theory, IV. On addition of finite sets, II, Elabužskiı Gosudarstvennyı̌ Pedagogičeskiǔ Institut. Učenye Zapiski 8 (1960), 72-116.

[25] G. Freiman, On the structure and the number of sum-free sets, Astérisque 209 (1992), 195-201.

[26] B. Green, The Cameron-Erdös conjecture, Bulletin of the London Mathematical Society 36 (2004), 769-778.

[27] B. Green, A Szemerédi-type regularity lemma in abelian groups, with applications, Geometric and Functional Analysis 15 (2005), 340-376.

[28] B. Green and I. Z. Ruzsa, Sum-free sets in abelian groups, Israel Journal of Mathematics 147 (2005), 157-189.

[29] B. Green and R. Morris, Counting sets with small sumset and applications, Combinatorica 36 (2016), 129-159.

[30] R. Hancock, K. Staden and A. Treglown, Independent sets in hypergraphs and Ramsey properties of graphs and the integers, arXiv:1701.04754.

[31] R. Hancock and A. Treglown, On solution-free sets of integers, European Journal of Combinatorics 66 (2017), 110-128.

[32] M. C. Hu, A note on sum-free sets of integers, Proceedings of the American Mathematical Society 80 (1980), 711-712.

[33] S. Janson, T. Łuczak and A. Ruciński, Random graphs, Wiley-Interscience Series in Discrete Mathematics and Optimization, Wiley-Interscience, New York, 2000.

[34] R. Jin, Freiman's inverse problem with small doubling property, Advances in Mathematics 216 (2007), 711-752.

[35] V. Lev, Large sum-free sets in ternary spaces, Journal of Combinatorial Theory. Series A 111 (2005), 337-346.

[36] V. Lev, Large sum-free sets in $\mathbb{Z} / p \mathbb{Z}$, Israel Journal of Mathematics 154 (2006), 221-234.

[37] V. Lev and P. Smeliansky, On addition of two distinct sets of integers, Acta Arithmetica 70 (1995), 85-91.

[38] T. Łuczak, On sum-free sets of natural numbers, Resenhas do Instituto de Matemática e Estatística da Universidade de São Paulo 2 (1995), 229-238.

[39] H. Plünnecke, Eine zahlentheoretische anwendung der graphtheorie, Journal für die Reine und Angewandte Mathematik 243 (1970), 171-183.

[40] I. Z. Ruzsa, Generalized arithmetical progressions and sumsets, Acta Mathematica Hungarica 65 (1994), 379-388.

[41] A. A. Sapozhenko, The Cameron-Erdös conjecture, Doklady Akademii Nauk 393 (2003), 749-752.

[42] D. Saxton and A. Thomason, Hypergraph containers, Inventiones Mathematicae 20 (2015), 925992.

[43] T. Tao and V. Vu, Additive Combinatorics, Cambridge Studies in Advanced Mathematics, Vol. 105, Cambridge University Press, 2006.

Department of Mathematics, ETH, 8092 Zurich

E-mail address: manh.tran@math.ethz.ch 